\title{
Reconfigurable Intelligent Surfaces for Localization: Position and Orientation Error Bounds
}

\author{
Ahmed Elzanaty, Member, IEEE, Anna Guerra, Member, IEEE, \\ Francesco Guidi, Member, IEEE, and Mohamed-Slim Alouini, Fellow, IEEE,
}

\begin{abstract}
Next-generation cellular networks will witness the creation of smart radio environments (SREs), where walls and objects can be coated with reconfigurable intelligent surfaces (RISs) to strengthen the communication and localization performance. In fact, RISs have been recently introduced not only to overcome communication blockages due to obstacles but also for high-precision localization of mobile users in GPS denied environments, e.g., indoors. Towards this vision, this paper presents the localization performance limits for communication scenarios where a single next generation NodeB base station (gNB), equipped with multiple-antennas, infers the position and the orientation of a user equipment (UE) in a RIS-assisted SRE. We consider a signal model that is valid also for near-field propagation conditions, as the usually adopted far-field assumption does not always hold, especially for large RISs. For the considered scenario, we derive the Cramér-Rao lower bound (CRLB) for assessing the ultimate localization and orientation performance of synchronous and asynchronous signalling schemes. In addition, we propose a closed-form RIS phase profile that well suits joint communication and localization, and we perform extensive numerical results to assess the performance of our scheme for various localization scenarios and for various RIS phase design. Numerical results show that the proposed scheme can achieve remarkable performance, even in asynchronous signalling, and that the proposed phase design, based on signal-to-noise ratio (SNR), approaches the numerical optimal phase design that minimizes the CRLB.
\end{abstract}

Index Terms-Reconfigurable intelligent surfaces, smart radio environment, single-anchor localization, attitude estimation, nearfield localization, Cramér-Rao lower bound.

\section{INTRODUCTION}

Recently, smart radio environments (SREs) have been conceived as a new paradigm where the traditional radio environment is turned into a smart reconfigurable space that plays an active role in transferring and processing the information [1], [2]. Indeed, key performance indicators (KPIs) for the next sixth generation mobile networks $(6 \mathrm{G})$ promote continuous connection availability, strong reliability, huge device density $\left(10^{7}\right.$ devices per $\left.\mathrm{km}^{2}\right)$ and air interface latency of sub-milliseconds (e.g., $10 \mu \mathrm{s}$ ), etc. [3], [4]. To meet these requirements, reconfigurable intelligent surfaces (RISs) might

A. Elzanaty and M.-S. Alouini are with King Abdullah University of Science and Technology (KAUST), Thuwal 23955-6900, Saudi Arabia, email: \{ahmed.elzanaty, slim.alouini\}@kaust.edu.sa. A. Elzanaty is also with the Department of Electrical and Computer Engineering, Sinai University, North Sinai 45518, Egypt. A. Guerra is with the WiLAB, University of Bologna, Italy, email: anna.guerra3@unibo.it. F. Guidi is with CNR-IEIIT, Italy, e-mail: francesco.guidi@ieiit.cnr.it. Anna Guerra thanks the support of the EU's H2020 research and innovation programme under the Marie Sklodowska-Curie project AirSens (793581). represent a key solution, allowing to enhance not only wireless communications but also imaging- and localization-based applications thanks to the augmented ambient awareness [4][6]. In this regard, RISs can aid in establishing a line-of-sight (LOS) link between the transmitter and the receiver even in the presence of obstructions or when the received power from the direct path does not enable a robust connection [7].

In analogy with software defined radios, RISs are often referred to as software defined surfaces (SDSs), where the electromagnetic response to the incident wave can be controlled by a software [8]. The realization of such a technology can be enabled by metamaterials, which are a class of artificial materials whose physical properties, such as permittivity and permeability, can be engineered to exhibit some desired characteristics [9]-[13]. When such metamaterials are deployed in metasurfaces, their effective parameters can be tailored to realize a desired transformation on the transmitted, received, or impinging waves [14]-[17]. With the availability of new degrees of freedom useful to improve the network performance, the environment will be no more perceived as a passive entity, but as a meaningful support for wireless communications based applications [18]-[23], e.g., energy transfer [24], vehicular networks [25], unmanned aerial vehicle (UAV) communications [26], physical layer security [24], cognitive radio [27], electromagnetic fields (EMF)-aware beamforming [28]-[30], and many others [31]. In this context, wireless localization with RISs [32], [33] has not yet received a large attention, albeit they represent a promising candidate for enhancing positioning and orientation estimation capabilities in next-generation cellular networks for various $6 \mathrm{G}$ applications, e.g., augmented reality and self-driving cars [25], [34]-[36]. This is of great help in GPS-denied environments, and it allows to avoid the use of a dedicated infrastructure, usually made of multiple anchors. Indeed, the possibility to localize with a single antenna array is not new, and has been investigated in the last few years [37]-[42]. In fact, the move-up in the frequency spectrum towards millimeter-waves (mm-wave) enables the integration of large number of antennas into small areas. By enabling such an array architecture, namely massive array, capable to realize near-pencil beam antennas, it becomes feasible not only to boost communication but also single-anchor localization capabilities at an unprecedented scale [43]-[48].

Current state-of-the-art for intelligent surfaces-based localization considers studies employing RIS either in receive mode [49] or in reflection mode [33], [50]. When exploited in receive mode, a large intelligent surface is used to localize a user in 
front of it, both in near-field and far-field [49], [51]. When instead operating in reflection mode, an approach exploiting the modification of the RIS reflection coefficient is proposed such that the experienced received signal strength (RSS) at different points is enlarged and the localization accuracy is improved [52]. Differently, in [33], authors exploit a RIS for supporting the positioning and communication in the $\mathrm{mm}$ wave frequency bands, assuming that the mobile is in farfield with respect to the RIS [33]. Such an approximation is not always valid, especially when large surfaces and arrays are considered with respect to the distance. Consequently, the entailed models are no more accurate, as the mobile is not in the Fraunhofer region but in the Fresnel region, where the wavefront has a considerable curvature, and it cannot be approximated as a plane wave. Additionally, ignoring the spherical wavefront discards essential information regarding the location and orientation of the mobile [53]-[55].

To the best of authors' knowledge, no paper has considered a general model accounting for 3D RIS-assisted localization and orientation estimation in near-field, as current papers for near-field positioning only refer to the adoption of a large intelligent surface in receive mode and not as a mean for controlling the multipath.

To this purpose, in this paper we consider the localization scenario depicted in Fig. 1, where we propose a localization scheme that makes use of infrastructures envisioned for next generation communication systems. Our model accounts for the incident spherical wavefront. Note that the user equipment (UE) can operate in the near-field region even for large distances provided that a large number of antennas is employed (e.g., with electrically large RIS) and the system operates at high frequency (e.g., millimeter-waves). Indeed, in SRE, the next generation NodeB base station (gNB) augments its environment awareness, as it allows also to achieve a knowledge of the environment in terms of inferring the location in the 3D space and the orientation (i.e., roll, pitch, and yaw) of the UE. In this context, we derive the Cramér-Rao lower bound (CRLB) to investigate the ultimate positioning and orientation estimation performance in the presence of the RIS. Then, we analyze the geometric dilution of precision (GDOP) to evaluate the impact of the geometry on the UE localization. Finally, we derive a suboptimal phase design for the RIS in closed-form to enhance both the localization and communication performance by maximizing the signal-to-noise ratio (SNR).

The main contributions can be summarized as follows.

- We propose an architecture where the RIS is used to assist mobile localization (position and orientation estimation) at the gNB, which can provide surveillance solutions or assist the communication process.

- We consider that the gNB, RIS, and the UE are equipped with multiple-antennas with arbitrary array configurations and geometries including planar arrays that will be adopted for beyond fifth generation mobile networks (5G) systems, especially the RIS, allowing 3D beamforming in both the azimuth and elevation, while most of the literature considers linear arrays with 2D beamforming that significantly simplifies the analysis to the conventional steering vectors.

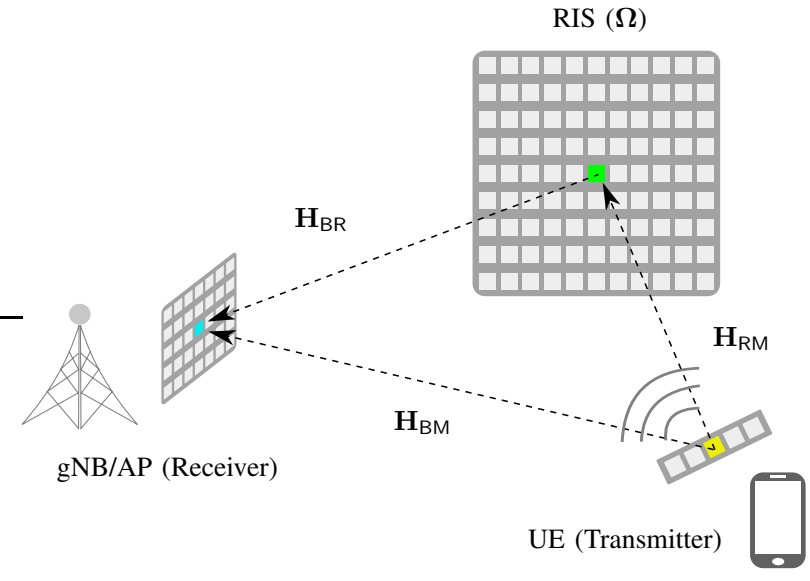

Fig. 1: Considered RIS-aided positioning scenario.

- We consider a general model valid for both near- and farfield localization and attitude (i.e., orientation) estimation in 3D space, unlike the literature that either imposes the far-field assumption or considers simplified $2 \mathrm{D}$ geometry.

- Differently from the state-of-the-art that analyzes synchronous systems, we consider two general signalling schemes (i.e., synchronous and asynchronous) and compare their localization error performance.

- We derive the ultimate bound on the localization performance in terms of the CRLB. Furthermore, to get more insights on the effect of the geometry (e.g., locations and orientations of the gNB, RIS, and UE) on the localization performance, we consider the GDOP metric.

- We propose a closed-form RIS phase design, that accounts for the spherical wavefront, and we compare it to other strategies along with various transmit beamforming techniques;

- We perform extensive simulations and numerical results that provide insights into the problem and shed light on the benefits offered by the adoption of the RIS in terms of localization performance.

The rest of the manuscript is organized as follows. Sec. II describes the signal model for both synchronous and asynchronous cases. Sec. III investigates the position and orientation performance limits and the impact of the system geometry on localization, whereas Sec. IV discusses a possible design for the RIS phase profile. In Sec. V simulation results are reported and final conclusions are drawn in Sec. VI.

Scalars (e.g., $x$ ) are denoted in italic, vectors (e.g., $\mathbf{x})$ in bold, and matrices (e.g., $\mathbf{X}$ ) in bold capital letters. $\nabla_{x}(a)=$ $\partial a / \partial x$ is the partial derivative of $a$ with respect to the scalar $x . \nabla_{\mathbf{x}}(\cdot)$ is the gradient operator with respect to the vector $\mathbf{x}$. Transpose and Hermitian operators are represented as ${ }^{\top}$ and ${ }^{\mathrm{H}}$, respectively. The $N \times N$ matrix with all elements being zeros and the $N \times N$ identity matrix are denoted by $\mathbf{0}_{N \times N}$ and $\mathbf{I}_{N \times N}$, respectively. The operator $\operatorname{tr}(\mathbf{X})$ denotes the trace of a matrix $\mathbf{X}$, while $\operatorname{diag}(\mathbf{x})$ denotes a diagonal matrix with diagonal elements identified by $\mathbf{x}$. A probability density function is denoted by $p(\cdot)$, and $\mathbb{E}\{\mathbf{x}\}$ is the expectation of a random vector $\mathbf{x}$ with respect to its distribution. $j=\sqrt{-1}$ is the imaginary unit. The operator $\|\cdot\|_{\ell}$ is the $\ell$-norm, and $\|\cdot\|$ 


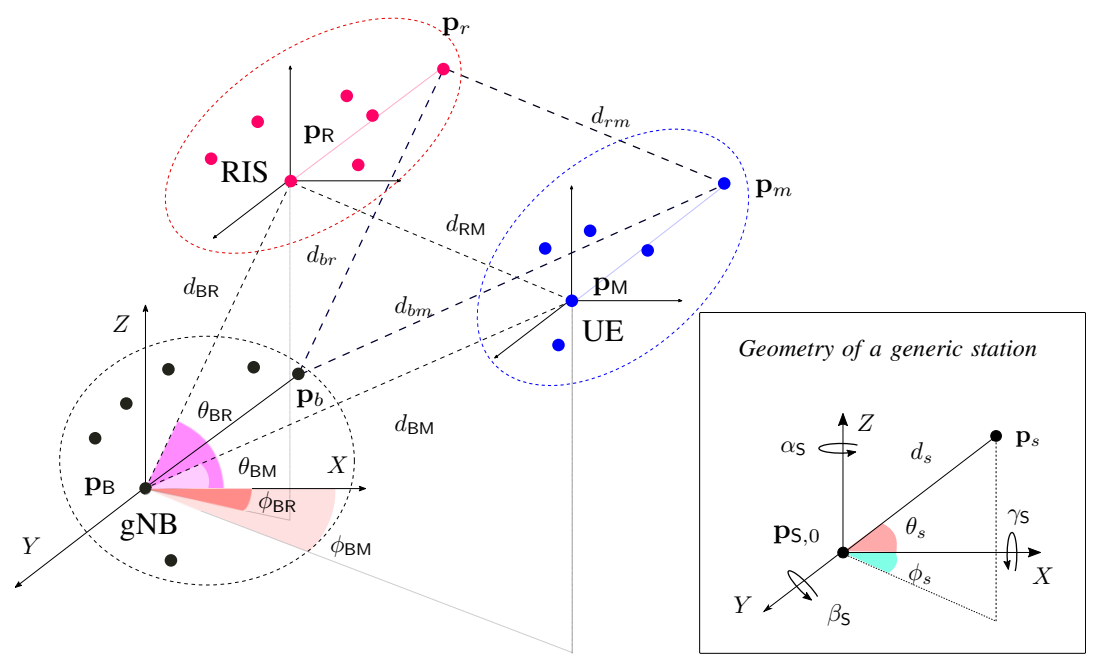

Fig. 2: 3D geometry of the considered localization scenario.

is the $\ell_{2}$-norm.

\section{SYSTEM MODEL}

\section{A. Localization Scenario}

In this paper, we consider a localization scenario as in Fig. 1 where a gNB, equipped with an antenna array with center located in position $\mathbf{p}_{\mathrm{B}}=\left[x_{\mathrm{B}}, y_{\mathrm{B}}, z_{\mathrm{B}}\right]^{\top}$, performs the position and orientation estimation of a UE with center in $\mathbf{p}_{\mathrm{M}}=\left[x_{\mathrm{M}}, y_{\mathrm{M}}, z_{\mathrm{M}}\right]^{\top}$ and rotated by $\phi_{\mathrm{M}}=\left[\alpha_{\mathrm{M}}, \beta_{\mathrm{M}}, \gamma_{\mathrm{M}}\right]^{\top}$. The geometry is reported in Fig. 2. The localization is aided by the presence of a RIS, with center located at a known position $\mathbf{p}_{\mathrm{R}}=\left[x_{\mathrm{R}}, y_{\mathrm{R}}, z_{\mathrm{R}}\right]^{\top}$, considered as a passive reflector that supports the gNB also for communicating with the UE.

According to Fig. 2 and considering the gNB as the center of the coordinate system, the UE and RIS centers' coordinates can be expressed as $\mathbf{p}_{\mathrm{S}} \triangleq\left[x_{\mathrm{S}}, y_{\mathrm{S}}, z_{\mathrm{S}}\right]^{\top}$ with $\mathrm{S} \in\{\mathrm{M}, \mathrm{R}\}$ being the label for a generic station and where the coordinates are

$$
\begin{aligned}
& x_{\mathrm{S}}=x_{\mathrm{B}}+d_{\mathrm{BS}} \cos \left(\theta_{\mathrm{BS}}\right) \cos \left(\phi_{\mathrm{BS}}\right), \\
& y_{\mathrm{S}}=y_{\mathrm{B}}+d_{\mathrm{BS}} \cos \left(\theta_{\mathrm{BS}}\right) \sin \left(\phi_{\mathrm{BS}}\right), \\
& z_{\mathrm{S}}=z_{\mathrm{B}}+d_{\mathrm{BS}} \sin \left(\theta_{\mathrm{BS}}\right) .
\end{aligned}
$$

Notably, the spherical coordinates can be easily retrieved from the equations above. Further, for each $S \in\{B, R, M\}$ and for each corresponding antenna index $s \in\{b, r, m\}$, we can indicate the antenna coordinates of each array as $\mathbf{p}_{\mathrm{S}, s}=\mathbf{p}_{s}=\left[x_{s}, y_{s}, z_{s}\right]^{\top}$ where $\forall s \in\left\{1,2, \cdots, N_{\mathrm{S}}\right\}$,

$$
\begin{aligned}
& x_{\mathrm{s}}=d_{s} \cos \left(\theta_{s}\right) \cos \left(\phi_{s}\right), \\
& y_{s}=d_{s} \cos \left(\theta_{s}\right) \sin \left(\phi_{s}\right), \\
& z_{s}=d_{s} \sin \left(\theta_{s}\right),
\end{aligned}
$$

where $N_{\mathrm{S}}$ is the number of antennas at the considered array, and $\phi_{s}$ and $\theta_{s}$ are the azimuth and elevation angles of the $s$-th antenna element measured from the array centroid, respectively. In addition, we consider arrays that can be rotated around the axes, that is $\forall s \in\left\{1,2, \cdots, N_{\mathrm{S}}\right\}$, we have

$$
\mathbf{p}_{\mathrm{S}, s}=\left[x_{\mathrm{S}, s}, y_{\mathrm{S}, s}, z_{\mathrm{S}, s}\right]^{\top}=\mathbf{R}\left(\alpha_{\mathrm{S}}, \beta_{\mathrm{S}}, \gamma_{\mathrm{S}}\right) \mathbf{p}_{\mathrm{S}, s}^{(0)} \text {, }
$$

with $\mathbf{p}_{\mathrm{S}, s}^{(0)}$ being the initial array deployment, and $\mathbf{R}\left(\alpha_{\mathrm{S}}, \beta_{\mathrm{S}}, \gamma_{\mathrm{S}}\right)$ is the rotation matrix given by the multiplication of the rotation matrices for each axis where $\left(\alpha_{S}, \beta_{S}, \gamma_{S}\right)$ are the yaw, pitch, and roll angles. The yaw corresponds to the azimuth, as it is the rotation around the $z$-axis and it is here indicated with $\alpha_{\mathrm{S}} . \beta_{\mathrm{S}}$ is the pitch, around the $y$-axis, whereas $\gamma_{\mathrm{S}}$ is the roll entailing a rotation around the $x$-axis. By considering counterclockwise rotations, the rotation matrix, $\mathbf{R}(\alpha, \beta, \gamma)$, is given in [56, (3.42)].

\section{B. Signal Model for Incident Spherical Wavefronts}

We now describe a model which accounts for spherical wavefront, and it is valid also for near-field propagation conditions. In the uplink, the UE transmits $N$ orthogonal frequency-division multiplexing (OFDM) subcarriers, i.e., for the $n$-th subcarrier with $n \in \mathbb{N}_{s} \triangleq\{1,2, \cdots, N\}$, we have

$$
\mathbf{x}[n]=\left[x_{1}, x_{2}, \ldots, x_{N_{\mathrm{M}}}\right]^{\top} \triangleq \mathbf{w}[n] p[n],
$$

where $N_{\mathrm{M}}$ is the number of antennas at the UE, $p[n]$ is the data symbol corresponding to the $n$-th subcarrier with $\mathbb{E}\left\{p[n] p^{*}[n]\right\}=1$, and $\mathbf{w}[n] \in \mathbb{C}^{N_{\mathrm{M}}}$ is the beamforming vector with $\|\mathbf{w}[n]\|^{2}=1$, which can be written as

$$
\mathbf{w}[n]=\left[a_{1} e^{j \beta_{1}}, a_{2} e^{j \beta_{2}}, \cdots, a_{m} e^{j \beta_{m}}, \cdots, a_{N_{\mathrm{M}}} e^{j \beta_{N_{\mathrm{M}}}}\right]^{\top},
$$

where $a_{m}$ and $\beta_{m}$ represent the $m$-th amplitude and phase of the transmit-beamformer. Let $\boldsymbol{\Theta} \triangleq\left\{\theta_{1}, \theta_{2}, \cdots, \theta_{N_{R}}\right\}$ be the vector containing the designed phase shifts induced at the RIS, and $N_{\mathrm{R}}$ is the number of RIS elements. Then, we indicate with

$$
\boldsymbol{\Omega}=\operatorname{diag}\left(e^{j \Theta}\right) \triangleq \operatorname{diag}\left(e^{j \theta_{1}}, e^{j \theta_{2}}, \ldots, e^{j \theta_{N_{\mathrm{R}}}}\right),
$$

the $N_{\mathrm{R}} \times N_{\mathrm{R}}$ diagonal matrix containing the RIS phases.

Differently from the RIS literature, the gNB estimates the UE position, $\mathrm{p}_{\mathrm{M}}$, and its orientation, $\phi_{\mathrm{M}}$, by exploiting the spherical waveform model. The received signal at the gNB for the $n$-th subcarrier can be written as [57]

$$
\begin{aligned}
\mathbf{y}[n] & =\sqrt{P} \mathbf{H}_{\mathrm{BM}}[n] \mathbf{x}[n]+\sqrt{P} \mathbf{H}_{\mathrm{BR}}[n] \boldsymbol{\Omega} \mathbf{H}_{\mathrm{RM}}[n] \mathbf{x}[n]+\boldsymbol{\omega}[n] \\
& \triangleq \boldsymbol{\mu}[n]+\boldsymbol{\omega}[n], \quad \forall n \in \mathbb{N}_{s}
\end{aligned}
$$


where $P$ is the signal power, $\mathbf{x}[n]$ is the transmitted vector for the $n$-th carrier frequency, $\boldsymbol{\omega}[n]$ is an additive thermal noise. The channel matrices are $\mathbf{H}_{\mathrm{BM}}[n], \mathbf{H}_{\mathrm{RM}}[n]$, and $\mathbf{H}_{\mathrm{BR}}[n]$ for the gNB-UE, RIS-UE, and gNB-RIS links of sizes $N_{\mathrm{B}} \times N_{\mathrm{M}}$, $N_{\mathrm{R}} \times N_{\mathrm{M}}$, and $N_{\mathrm{B}} \times N_{\mathrm{R}}$, respectively. In the following, we discriminate whether the gNB and the UE have been synchronized or not. For non-synchronous systems, the position information can still be gathered from the spherical wavefront, even if no information can be retrieved from the time-of-arrival (TOA).

1) Synchronous System: We here consider that a synchronization procedure has been performed between the gNB and the UE prior the localization step. Once synchronized, the positioning information can be retrieved by jointly processing temporal and angular information of the received signal. By extending (10) to its scalar notation, the general model of the received signal can be rewritten as

$$
y_{b}[n]=\mu_{b}[n]+w[n], \quad \forall b \in\left\{1,2, \cdots, N_{\mathrm{B}}\right\},
$$

with $N_{\mathrm{B}}$ being the number of antennas at the gNB and $w[n]$ being the circularly symmetric zero-mean Gaussian noise with power spectral density $\sigma^{2}$. The useful part of the signal, without the noise, is

$$
\begin{aligned}
\mu_{b}[n] \triangleq & \sqrt{P} \sum_{m=1}^{N_{\mathrm{M}}} x_{m}[n] e^{-j 2 \pi f_{n} \xi_{\mathrm{BM}}}\left(\rho_{\mathrm{BM}} e^{-j 2 \pi f_{n}\left(\tau_{b m}+\eta_{m}\right)}\right. \\
& \left.+\rho_{\mathrm{BRM}} \sum_{r=1}^{N_{\mathrm{R}}} e^{j \theta_{r}} e^{-j 2 \pi f_{n}\left(\tau_{b r}+\tau_{r m}+\eta_{r}+\eta_{m}\right)}\right),
\end{aligned}
$$

where $f_{n}=f_{\mathrm{c}}+n \Delta f-B / 2$ is the frequency of the $n$-th subcarrier, $f_{\mathrm{c}}$ is the carrier frequency, $\Delta f$ is the subcarrier spacing, $B=N \Delta f$ is the signal bandwidth, and $\tau_{b m}, \tau_{b r}$, $\tau_{r m}$ are the delays for each couple of antenna (e.g., $\tau_{b m}$ is the delay between the $b$-th antenna at the gNB and the $m$-th antenna at the UE), $\xi_{\mathrm{BM}}$ is a synchronization residual (negligible after accurate synchronization procedures), and $\eta_{m}$ and $\eta_{r}$ are array non-idealities. The signal attenuation coefficients due to propagation are indicated with $\rho_{\mathrm{BM}}$ and $\rho_{\text {BRM }}$ for the direct and the relayed path, respectively. ${ }^{1}$ Since the plane-wave approximation is not always valid, a spherical model is considered where the following relations hold

$$
\begin{aligned}
& \tau_{b m}\left(d_{\mathrm{BM}}, \theta_{\mathrm{BM}}, \phi_{\mathrm{BM}}\right)=d_{b m} / c, \\
& \tau_{b r}\left(d_{\mathrm{BR}}, \theta_{\mathrm{BR}}, \phi_{\mathrm{BR}}\right)=d_{b r} / c, \\
& \tau_{r m}\left(d_{\mathrm{RM}}, \theta_{\mathrm{RM}}, \phi_{\mathrm{RM}}\right)=d_{r m} / c, \\
& \rho_{\mathrm{BM}}=\frac{\lambda}{4 \pi} \frac{1}{d_{\mathrm{BM}}}, \\
& \rho_{\mathrm{BRM}}=\frac{\lambda}{4 \pi} \frac{1}{d_{\mathrm{RM}}+d_{\mathrm{BR}}},
\end{aligned}
$$

where $c$ is the speed of light, $\left(d_{\mathrm{BM}}, \theta_{\mathrm{BM}}, \phi_{\mathrm{BM}}\right)$, $\left(d_{\mathrm{BR}}, \theta_{\mathrm{BR}}, \phi_{\mathrm{BR}}\right)$ and $\left(d_{\mathrm{RM}}, \theta_{\mathrm{RM}}, \phi_{\mathrm{RM}}\right)$ are the distances

\footnotetext{
${ }^{1}$ According to the considered system geometry, the signal amplitude is about the same at each antenna as its variations are negligible.
}

and angles between the gNB-UE, gNB-RIS, RIS-UE centroids, respectively, and where

$$
d_{b m}=\sqrt{d_{m}^{2}+d_{b}^{2}+d_{\mathrm{BM}}^{2}-2\left(\mathrm{G}_{b m}^{(1)}+d_{\mathrm{BM}} \mathrm{G}_{b m}^{(2)}\right)},
$$

with $\mathrm{G}_{b m}^{(1)}$ and $\mathrm{G}_{b m}^{(2)}$ containing the information of the geometry at the transmitter and at the receiver, that is

$$
\begin{aligned}
\mathrm{G}_{b m}^{(1)} & =x_{b} x_{m}+y_{b} y_{m}+z_{b} z_{m} \\
\mathrm{G}_{b m}^{(2)} & =\left(x_{m}-x_{b}\right) \cos \theta_{\mathrm{BM}} \cos \phi_{\mathrm{BM}}+\left(y_{m}-y_{b}\right) \\
& \times \cos \theta_{\mathrm{BM}} \sin \phi_{\mathrm{BM}}+\left(z_{m}-z_{b}\right) \sin \theta_{\mathrm{BM}} .
\end{aligned}
$$

The distances of arrival between the $b$-th gNB antenna and the $r$-th RIS antenna and between the $r$ th RIS antenna and the $m$ th UE antenna, namely $d_{b r}$ and $d_{r m}$, can be found using (18) with appropriate substitutions, as done in (1) and (4).

Differently from traditional schemes that make the assumption of incident planar wavefront, in (18) we infer jointly the ranging and bearing information from the spherical waveform curvature. Notably, it is possible to write (12) only when the clocks of the gNB, RIS and UE have been synchronized. The accurate synchronization might entail several and long procedures. In the following, we consider an asynchronous alternative where it is still possible to retrieve the UE position from the relative phases.

2) Asynchronous System: As evidenced in (11), from the received signal it is possible to infer the TOA estimate, which is possible in all those situations where a synchronization procedure has been performed. In this case, the system is no more able to directly estimate the information of the distance from the TOA. Instead, the incident waveform curvature, i.e.,

$$
\begin{aligned}
& \Delta d_{b m}=d_{b m}-d_{\mathrm{BM}}=c \Delta \tau_{b m}, \\
& \Delta d_{b r}=d_{b r}-d_{\mathrm{BR}}=c \Delta \tau_{b r},
\end{aligned}
$$

can be exploited for UE localization.

$$
\begin{array}{r}
y_{b}[n]=\sqrt{P} \sum_{m=1}^{N_{\mathrm{M}}} x_{m}[n]\left(\rho_{\mathrm{BM}} e^{-j \chi_{\mathrm{BM}}} e^{-j 2 \pi f_{n}\left(\Delta \tau_{b m}+\eta_{m}\right)}\right. \\
\left.+\rho_{\mathrm{BRM}} e^{-j \chi_{\mathrm{BRM}}} \sum_{r=1}^{N_{\mathrm{R}}} e^{j \theta_{r}} e^{-j 2 \pi f_{n}\left(\Delta \tau_{b r}+\Delta \tau_{r m}+\eta_{r}+\eta_{m}\right)}\right) \\
+w[n],
\end{array}
$$

where $\chi_{\mathrm{BM}}$ and $\chi_{\mathrm{BRM}}$ are uniformly distributed random variable from 0 to $2 \pi$ representing the phase offsets between the $\mathrm{gNB}$, the UE and the RIS due to the lack of synchronization.

Given the proposed models for synchronous and asynchronous systems, in the following we derive the attainable fundamental performance limits.

\section{RIS-AIDED POSITION AND ORIENTATION ERROR BOUNDS}

In this section, we derive the performance limits for the considered localization scenario in the free-space LOS direct and reflective RIS scenario, where the ultimate localization limits can be rigorously derived. To this end, the CRLB is a useful metric that represents the minimum variance of the 
estimation error from any unbiased estimator, and it can be represented with the inverse of the Fisher information matrix (FIM) [58], [59]. Then, we investigate the impact of the geometry on the error through the GDOP metric analysis.

\section{A. The CRLB on UE Position and Orientation}

Given the signal models in (11) and (23), we distinguish two possible estimation approaches: $(i)$ the first one exploits a direct localization approach, and it is used for the asynchronous case; (ii) the second one is a two-stage approach that considers that the location and orientation are estimated from a set of features extracted from the signal, e.g., TOAs, angle-of-arrivals (AOAs), and received signal strength indicators (RSSIs) [60]. In both cases, the parameter vector to be estimated is

$$
\mathbf{s}=\left[\mathbf{p}_{\mathrm{M}}, \boldsymbol{\phi}_{\mathrm{M}}\right]^{\mathrm{T}},
$$

where $\mathbf{p}_{M} \in \mathbb{R}^{3}$, and $\phi_{M} \in[0,2 \pi]^{3}$ contain the UE position and orientation parameters, as indicated in Section II-A. On the other hand, the measurement vector can be written as

$$
\begin{aligned}
& \boldsymbol{\Gamma}=\mathbf{s}, \\
& \boldsymbol{\Gamma}=\left[\rho_{\mathrm{BM}}, \theta_{\mathrm{BM}}, \phi_{\mathrm{BM}}, \tau_{\mathrm{BM}}, \rho_{\mathrm{BRM}}, \theta_{\mathrm{RM}}, \phi_{\mathrm{RM}}, \tau_{\mathrm{RM}}, \phi_{\mathrm{M}}\right]^{\top},
\end{aligned}
$$

for the Synchronous and Asynchronous signalling, respectively, where $\tau_{\mathrm{BM}}$ and $\tau_{\mathrm{RM}}, \rho_{\mathrm{BM}}$ and $\rho_{\mathrm{BRM}}$, and $\theta_{\mathrm{BM}}, \phi_{\mathrm{BM}}, \theta_{\mathrm{RM}}$ $\phi_{\mathrm{RM}}$ are the TOAs, RSSI, and AOAs, respectively. All the main parameters that are required to infer the location and orientation of the UE are included in (26). ${ }^{2}$

The first approach with the measurement parameter vector expressed in (25) will be also referred to as "Direct" because it directly estimates the state. On the other side, the second approach in (26) will be also named "Two-stage" because it uses intermediate parameters to infer the UE position and orientation. Note that the two approaches are the same from a CRLB perspective. Still, we distinguish between the two parameter vectors for synchronous and asynchronous signalling.

This can be ascribed to a twofold reason: $(i)$ The vector of measurements in the synchronous case allows to emphasize the parameters of the received signal which depend on the position and orientation and to quantify the error in estimating these parameters; (ii) On the contrary, if a two-stage approach is used in the asynchronous case, where only difference of TOAs are present in (21), then the measurement vector would consist of all the TOA pairs between the gNB-UE and RISUE, leading to a dimensional issue for the FIM. Thus, a direct approach is adopted in which the position is directly inferred from the signals received at each antenna of the gNB, allowing the measurement vector to be written in a more compact way.

Starting from (25)-(26), the CRLB on the UE state vector can be written from $[58,(178)]$ as

$$
\mathbf{\Lambda}(\mathbf{s}) \triangleq\left[\sum_{n=1}^{N} \mathbf{I}_{n}(\mathbf{s})\right]^{-1}
$$

${ }^{2}$ If the localization is only based on TOA and AOAs, then the parameters related to RSSI (i.e., $\rho_{\mathrm{BM}}$ and $\rho_{\mathrm{BRM}}$ ) can be neglected in (26). Nevertheless, the resulting bound will be an upper bound on the CRLB derived directly from the signal. where $\mathbf{I}_{n}(\mathbf{s})$ is the FIM of the state vector relative to the $n$-th subcarrier. Hence, the position error bound (PEB) and orientation error bound (OEB) can be written as

$$
\mathrm{PEB}=\sqrt{\operatorname{tr}\left([\boldsymbol{\Lambda}(\mathbf{s})]_{1: 3,1: 3}\right)}, \quad \text { OEB }=\sqrt{\operatorname{tr}\left([\boldsymbol{\Lambda}(\mathbf{s})]_{4: 6,4: 6}\right)},
$$

where $[\cdot]_{a: b, c: d}$ indicates the sub-matrix located between rows $(a, b)$ and columns $(c, d)$.

The FIM can be obtained by the chain rule as [61]

$$
\mathbf{I}_{n}(\mathbf{s})=\left(\nabla_{\mathbf{s}} \boldsymbol{\Gamma}\right) \mathbf{I}_{n}(\boldsymbol{\Gamma})\left(\nabla_{\mathbf{s}} \boldsymbol{\Gamma}\right)^{\top},
$$

where $\mathbf{I}_{n}(\boldsymbol{\Gamma})$ is the FIM of the parameter vector in (25), i.e.,

$$
\mathbf{I}_{n}(\boldsymbol{\Gamma})=\mathbb{E}\left\{\left(\nabla_{\boldsymbol{\Gamma}} \log p(\mathbf{y}[n] ; \boldsymbol{\Gamma})\right)^{\mathrm{H}} \nabla_{\boldsymbol{\Gamma}} \log p(\mathbf{y}[n] ; \boldsymbol{\Gamma})\right\},
$$

with $\mathbf{J} \triangleq \nabla_{\mathbf{s}} \boldsymbol{\Gamma}$ being the Jacobian matrix and $\log p(\mathbf{y}[n] ; \boldsymbol{\Gamma})$ is the log-likelihood function of the received signal vector [58]. The log-likelihood function is computed from (11) as

$$
\begin{aligned}
\log p(\mathbf{y}[n] ; \boldsymbol{\Gamma})= & -(\mathbf{y}[n]-\boldsymbol{\mu}[n])^{\mathbf{H}} \boldsymbol{\Sigma}^{-1}(\mathbf{y}[n]-\boldsymbol{\mu}[n]) \\
& -N_{\mathrm{B}} \log \left(\pi \sigma^{2}\right),
\end{aligned}
$$

where $\boldsymbol{\Sigma}=\sigma^{2} \mathbf{I}_{N_{\mathrm{B}} \times N_{\mathrm{B}}}$ is the covariance matrix of the noise. The Jacobian matrix can be written as

$$
\begin{array}{lll}
\mathbf{J} & =\mathbf{I}_{6 \times 6}, & \text { Synchronous case } \\
\mathbf{J} & =\left[\begin{array}{cc}
\mathbf{J}_{\mathbf{p}_{\mathbf{M}}} & \mathbf{0}_{3 \times 3} \\
\mathbf{0}_{3 \times 3} & \mathbf{I}_{3 \times 3},
\end{array}\right], & \text { Asynchronous case }
\end{array}
$$

with $\mathbf{J}_{\mathbf{p}_{\mathbf{M}}}$ indicating the term relative to the UE position, and it is given in the Appendix A.

The elements of the FIM in (30) can be written as [61]

$$
\left[\mathbf{I}_{n}(\boldsymbol{\Gamma})\right]_{i, j}=\frac{2}{\sigma^{2}} \operatorname{Re}\left\{\sum_{b=1}^{N_{\mathrm{B}}} \frac{\partial \mu_{b}^{*}[n]}{\partial \Gamma_{i}} \frac{\partial \mu_{b}[n]}{\partial \Gamma_{j}}\right\}
$$

and their derivations are in Appendix B.

Since we are considering the curvature of the wavefront in (18), the bound is valid also for the near-field localization that is essential when the size of arrays are sufficiently large, within the Fraunhofer distance [62]. Also, the CRLB in (27) accounts for the errors due to the receiver noise (i.e., in $\sigma^{2}$ ) and the geometry of the localization scenario.

\section{B. Localization Algorithm}

One possible solution for estimating the location and orientation of the UE is through the maximum likelihood estimator (MLE). The UE location and orientation, $\mathbf{s}$, that maximize the log-likelihood function in (31) can be estimated as

$$
\widehat{\mathbf{s}} \triangleq \underset{\mathbf{p}_{\mathrm{M}} \in \mathbb{R}^{3}, \boldsymbol{\phi}_{\mathrm{M}} \in[0,2 \pi]^{3}}{\operatorname{argmax}} \log p(\mathbf{y}[n] ; \mathbf{s}) .
$$

The previous optimization problem can be solved by a grid search or by iterative methods such as Newton-Raphson and expectation-maximization algorithms. However, the convergence of iterative methods to the global maximum is not guaranteed.

The MLE is known to approach the CRLB derived in Section III-A for asymptotically high SNRs. Indeed, if there 
exists an efficient estimator with a variance that coincides with the CRLB, it will be the MLE, which can be found by simultaneously solving the following equations [61]

$$
\nabla_{\mathbf{s}} p(\mathbf{y}[n] ; \mathbf{s})=\mathbf{0}_{6 \times 1} \text {. }
$$

For asymptotically large number of measurements, i.e., snapshots $\mathbf{y}$ in (10), or high SNR, the error in estimating the location and orientation tends in distribution to a zero mean Gaussian distribution (indicated with "d") with covariance matrix $\boldsymbol{\Lambda}(\mathbf{s})$ expressed in (27), i.e.,

$$
\widehat{\mathbf{s}}-\mathbf{s} \stackrel{\mathrm{d}}{\longrightarrow} \mathcal{N}\left(\mathbf{0}_{6 \times 1}, \boldsymbol{\Lambda}(\mathbf{s})\right),
$$

where $\widehat{\mathbf{s}}$ is a random vector representing the estimated parameters through the MLE [61].

Alternatively, the two-stage approach can be adopted, where the signal attenuation coefficients can be estimated from the RSSI. The direction of arrival (i.e., AOAs) can be infered through variations of MUSIC algorithms or compressive sensing for both on- and off-grid methods with guaranteed recovery under some mild conditions. The compressive sensing based estimators have the advantage that the angles can be recovered even from a single snapshot of $\mathbf{y}$, while the performance can be further enhanced by considering multiple measurement vectors (i.e., several snapshots) [63]-[66]. For TOA estimation, two possible schemes can be considered based on correlators (i.e., matched filters) or on energy-based solutions (i.e., energy detectors) [67]-[70]. The second is more practical as it can operate at sub-Nyquist rate.

\section{Geometry Impact on Direct RIS-aided Localization}

The CRLB derived in Sec. III-A does not explicitly quantify the impact of the system geometry on the performance, because it includes also the effect of the input noise [71]. Hence, we now investigate the solely impact of the geometry on the localization performance using a GDOP analysis. In particular, as a GDOP metric, we consider the ratio between the root mean square error (RMSE) of position and the RMSE of measurement (ranging) error, i.e., [72]

$$
\mathrm{GDOP}=\frac{\sqrt{\sigma_{x}^{2}+\sigma_{y}^{2}+\sigma_{z}^{2}}}{\sigma_{M}} \triangleq \frac{\operatorname{RMSE}(\mathbf{p})}{\sigma_{M}}
$$

where $\sigma_{M}$ is the RMSE of the measurements, e.g., in GPS positioning it is the standard deviation of ranging measurements. Since the RMSE is lower bounded by the CRLB, the GDOP can be also defined as a function of the PEB as

$$
\sigma_{M} \cdot \mathrm{GDOP}=\operatorname{RMSE}(\mathbf{p}) \geq \sqrt{\operatorname{tr}(\mathrm{CRB}(\mathbf{p}))}=\mathrm{PEB} .
$$

Differently from the parameter vector in (25), in direct localization, position and orientation are directly estimated from the received signals at the receiver, with $\Gamma=\mathbf{s}$ as in (26). In this specific case, the measurement noise standard deviation is the same for all the antennas and corresponds to the thermal noise, i.e. $\sigma_{M}=\sigma$.
Given such signal-related measurements, the GDOP can be computed from the CRLB expression in (27) where the generic element in the FIM is given by

$$
\left[\mathbf{I}_{n}(\mathbf{s})\right]_{i, j}=\frac{2}{\sigma^{2}} \operatorname{Re}\left\{\sum_{b=1}^{N_{\mathrm{B}}} \frac{\partial \mu_{b}^{*}[n]}{\partial \ell_{i}} \frac{\partial \mu_{b}[n]}{\partial \ell_{j}}\right\},
$$

where $\ell_{i} \in \mathbf{s}$ is either related to the position or to the orientation of the UE. Therefore, we can write the GDOP for position and orientation as [72]

$$
\begin{aligned}
& \operatorname{GDOP}_{\mathbf{p}_{\mathrm{M}}}=\frac{1}{\sigma \kappa_{\mathbf{p}_{\mathrm{M}}}} \sqrt{\operatorname{tr}\left(\left[\sum_{n=1}^{N} \mathbf{I}_{n}(\mathbf{s})\right]_{1: 3,1: 3}^{-1}\right)}=\frac{\mathrm{PEB}}{\sigma \kappa_{\mathbf{p}_{\mathrm{M}}}}, \\
& \operatorname{GDOP}_{\boldsymbol{\phi}_{\mathrm{M}}}=\frac{1}{\sigma \kappa_{\boldsymbol{\phi}_{\mathrm{M}}}} \sqrt{\operatorname{tr}\left(\left[\sum_{n=1}^{N} \mathbf{I}_{n}(\mathbf{s})\right]_{4: 6,4: 6}^{-1}\right)}=\frac{\mathrm{OEB}}{\sigma \kappa_{\boldsymbol{\phi}_{\mathrm{M}}}}
\end{aligned}
$$

where $\kappa_{\mathbf{p}_{\mathrm{M}}}[\mathrm{m} / \sqrt{\mathrm{Watt}}]$ and $\kappa_{\boldsymbol{\phi}_{\mathrm{M}}}[$ radians $/ \sqrt{\text { Watt }}]$ are the normalization factors for the GDOP to become dimensionless. For example, in our settings the normalization factors $\kappa_{\mathbf{p}_{\mathrm{M}}}$ and $\kappa_{\phi_{\mathrm{M}}}$ can be designed as $d_{\mathrm{BM}} / \sqrt{P}$ and $1 / \sqrt{P}$, respectively. With this definition, the position and orientation errors are proportional to the GDOP, i.e., PEB $\propto \sigma \mathrm{GDOP}_{\mathbf{p}_{\mathrm{M}}}$ and $\mathrm{OEB} \propto \sigma \mathrm{GDOP}_{\phi_{\mathrm{M}}}[72]$.

\section{RIS PhASE DESIGN}

In order to enhance the performance of the proposed architecture and to exploit as much as possible the RIS potentialities, a proper joint design of the RIS phase profile and of the beamformer is essential. Unfortunately, the literature usually entails planar wavefronts incident to the RIS [36].

Thus, in the following we consider possible alternatives for the design of the RIS phase profile and transmit-beamformers accounting for spherical wavefronts.

1) Optimal RIS Phase Design: The first possibility considers the optimal phase shifts induced at the RIS and beamformers that minimize the position or orientation error bounds, i.e., we solve the following optimization programs

$$
\begin{array}{cr}
\text { Minimize } & \operatorname{PEB}(\boldsymbol{\Theta}, \mathbf{W}), \\
\boldsymbol{\Theta} \in[0,2 \pi]^{N_{\mathrm{R}}}, \mathbf{W} \in \mathbb{C}^{N_{\mathrm{M}} \times N} & \\
\text { Subject to } & \|\mathbf{w}[n]\|^{2}=1, \forall n \in \mathbb{N}_{s} \\
\begin{array}{c}
\text { Minimize } \\
\Theta \in[0,2 \pi]^{N_{\mathrm{R}}}, \mathbf{W} \in \mathbb{C}^{N_{\mathrm{M}} \times N}
\end{array} & \operatorname{OEB}(\boldsymbol{\Theta}, \mathbf{W}), \\
\text { Subject to } & \|\mathbf{w}[n]\|^{2}=1, \forall n \in \mathbb{N}_{s},
\end{array}
$$

where $\mathbf{W}$ is a matrix whose $n$-th column is $\mathbf{w}[n]$. Unfortunately, the optimization of the phase design and of the beamforming vectors is quite complex for two main reasons: (i) the number of real optimization variables, i.e, $N_{\mathrm{R}}+2 N_{\mathrm{M}} N$, is large; (ii) the joint optimization of coupled optimization variables, i.e., RIS phases and beamformers, usually entails non-convexity [73].

Concerning the first point $(i)$, we can consider the same beamforming vector for all subcarriers so that the number of optimization variables is reduced to $N_{\mathrm{R}}+2 N_{\mathrm{M}}$, under the assumption that $f_{\mathrm{c}} \gg B$, which is generally valid for mm-waves. 
We can further halve the number of beamforming optimization variables by optimizing only the phases of the beamformer, at the expense of some degradation in performance.

Concerning the second point (ii), alternate optimization (AO) can be considered to decouple the beamformer and the phase profile [73]. In this method, the phase profile is optimized for a fixed beamforming vector and vice versa until the convergence is reached.

Notably, if from one side this approach is complex as it involves the minimization of the inverse of the FIM, from the other side it represents the optimal solution in the sense of minimizing the position or orientation error bounds.

2) Proposed RIS Phase Design: Another possibility is to consider an ad-hoc approach that maximizes the sum of the SNRs for the central subcarrier at each gNB antenna as

$$
\begin{array}{cc}
\underset{\Theta \in[0,2 \pi]^{N_{R}}, \mathbf{w} \in \mathbb{C}^{N_{M} \times 1}}{\operatorname{Maximize}} & \operatorname{SNR}(\boldsymbol{\Theta}, \mathbf{w}), \\
\text { Subject to } & \|\mathbf{w}\|^{2}=1,
\end{array}
$$

where

$$
\begin{aligned}
\operatorname{SNR}(\boldsymbol{\Theta})= & \frac{P}{\sigma^{2}}\left\|\mathbf{H}_{\mathrm{BM}} \mathbf{w}+\mathbf{H}_{\mathrm{BR}} \boldsymbol{\Omega}(\boldsymbol{\Theta}) \mathbf{H}_{\mathrm{RM}} \mathbf{w}\right\|^{2} \\
\leq & \frac{P}{\sigma^{2}}\left(\left\|\mathbf{H}_{\mathrm{BM}} \mathbf{w}\right\|^{2}+\left\|\mathbf{H}_{\mathrm{BR}} \boldsymbol{\Omega}(\boldsymbol{\Theta}) \mathbf{H}_{\mathrm{RM}} \mathbf{w}\right\|^{2}\right. \\
& \left.+2\left\|\mathbf{H}_{\mathrm{BM}} \mathbf{w} \odot \mathbf{H}_{\mathrm{BR}} \boldsymbol{\Omega}(\boldsymbol{\Theta}) \mathbf{H}_{\mathrm{RM}} \mathbf{w}\right\|_{1}\right),
\end{aligned}
$$

with $\odot$ being the Hadamard element-wise product. Note that (46) results from the Cauchy-Schwarz inequality, where the equality holds only if the phase of the direct path coincides with the phase of the reflected path.

In order to design the RIS phase profile, we operate as follows: (i) first, we maximize the upper bound on the SNR in (46); (ii) then, we design an additional constant phase shift for the RIS phase profile such that the Cauchy-Schwartz inequality is tight, that is, the direct and the reflective link are almost coherently summed up at each antenna.

According to the aforementioned considerations, for a fixed beamforming vector $\mathbf{w}$, the RIS phase profile can be designed to maximize the upper bound on the SNR in (46). Nevertheless, the coupling between the direct and the reflective channels complicates the optimization. In this regard, we relax the optimization problem by ignoring the coupling and maximizing only the sum of the SNRs for the direct and RIS reflected links, i.e.,

$$
\underset{\boldsymbol{\Theta} \in[0,2 \pi]^{N_{\mathrm{R}}}}{\operatorname{Maximize}} \frac{P}{\sigma^{2}}\left(\left\|\mathbf{H}_{\mathrm{BM}} \mathbf{w}\right\|^{2}+\left\|\mathbf{H}_{\mathrm{BR}} \boldsymbol{\Omega}(\boldsymbol{\Theta}) \mathbf{H}_{\mathrm{RM}} \mathbf{w}\right\|^{2}\right) .
$$

The optimization can be further simplified by ignoring terms that does not depend on $\Theta$, then we have

$$
\underset{\boldsymbol{\Theta} \in[0,2 \pi]^{N_{\mathrm{R}}}}{\operatorname{Maximize}}\left\|\mathbf{H}_{\mathrm{BR}} \boldsymbol{\Omega}(\boldsymbol{\Theta}) \mathbf{H}_{\mathrm{RM}} \mathbf{w}\right\|^{2},
$$

that gives

$$
\underset{\Theta \in[0,2 \pi]^{N_{\mathrm{R}}}}{\operatorname{Maximize}} \sum_{b=1}^{N_{\mathrm{B}}}\left|\sum_{r=1}^{N_{\mathrm{R}}} \sum_{m=1}^{N_{\mathrm{M}}} a_{m} e^{j \beta_{m}} e^{j \theta_{r}} e^{-j 2 \pi f_{\mathrm{c}}\left(\tau_{b r}+\tau_{r m}\right)}\right|^{2} .
$$

Unfortunately, the number of degrees of freedoms, i.e., the number of controllable phase shifts at the RIS, is not enough to perfectly adjust the phase of the signals at the gNB. To combat such an issue, we relax the problem by minimizing the sum of square distance of the phases from their related centroid $\bar{\phi}(\boldsymbol{\Theta})$, inspired by the $K$-means algorithm [74]. Thus, we write

$$
\begin{aligned}
\underset{\boldsymbol{\Theta} \in[0,2 \pi]^{N_{\mathrm{R}}}}{\operatorname{Minimize}} \gamma(\boldsymbol{\Theta}) \triangleq & \sum_{r=1}^{N_{\mathrm{R}}} \sum_{b=1}^{N_{\mathrm{B}}} \sum_{m=1}^{N_{\mathrm{M}}}\left[\theta_{r}+\beta_{m}\right. \\
& \left.-2 \pi f_{\mathrm{c}}\left(\tau_{b r}+\tau_{r m}\right)-\bar{\phi}(\boldsymbol{\Theta})\right]^{2},
\end{aligned}
$$

where $\gamma(\boldsymbol{\Theta})$ is the objective function of interest, and the centroid $\bar{\phi}(\mathbf{\Theta})$ is given by

$$
\begin{aligned}
& \bar{\phi}(\boldsymbol{\Theta})=\frac{1}{N_{\mathrm{R}} N_{\mathrm{M}} N_{\mathrm{B}}} \sum_{r=1}^{N_{\mathrm{R}}} \sum_{b=1}^{N_{\mathrm{B}}} \sum_{m=1}^{N_{\mathrm{M}}}\left[\theta_{r}+\beta_{m}-2 \pi f_{\mathrm{c}}\left(\tau_{b r}+\tau_{r m}\right)\right] \\
& =\frac{1}{N_{\mathrm{R}}} \theta_{k}+\frac{1}{N_{\mathrm{R}}} \sum_{r=1, r \neq k}^{N_{\mathrm{R}}} \theta_{r}+\frac{1}{N_{\mathrm{M}} N_{\mathrm{B}} N_{\mathrm{R}}} \sum_{r=1}^{N_{\mathrm{R}}} \sum_{b=1}^{N_{\mathrm{B}}} \sum_{m=1}^{N_{\mathrm{M}}} C_{b r m},
\end{aligned}
$$

where $C_{b r m}=\beta_{m}-2 \pi f_{c}\left(\tau_{b r}+\tau_{r m}\right)$. It can be verified that $\gamma(\boldsymbol{\Theta})$ is a convex function. More precisely, $\gamma(\boldsymbol{\Theta})$ is convex, as the composition of a convex function with an affine mapping is convex, and the positive weighted sum of convex functions preserves the function convexity $[75,3.2 .2]$ and $[75,3.2 .1]$.

For $k \in \mathbb{K} \triangleq\left\{1,2, \cdots, N_{\mathrm{R}}\right\}$, the objective function can be expressed as

$$
\begin{aligned}
\gamma(\boldsymbol{\Theta})= & \sum_{b=1}^{N_{\mathrm{B}}} \sum_{m=1}^{N_{\mathrm{M}}}\left[\theta_{k}+C_{b k m}-\bar{\phi}(\boldsymbol{\Theta})\right]^{2} \\
& +\sum_{r=1, r \neq k}^{N_{\mathrm{R}}} \sum_{b=1}^{N_{\mathrm{B}}} \sum_{m=1}^{N_{\mathrm{M}}}\left[\theta_{r}+C_{b r m}-\bar{\phi}(\boldsymbol{\Theta})\right]^{2} .
\end{aligned}
$$

Since the objective function is convex, the optimal solution can be found by solving the following equations in $\theta_{k}$ [75],

$$
\begin{aligned}
& \frac{\partial \gamma(\boldsymbol{\Theta})}{\partial \theta_{k}}=\sum_{b=1}^{N_{\mathrm{B}}} \sum_{m=1}^{N_{\mathrm{M}}} 2\left(1-\frac{1}{N_{\mathrm{R}}}\right)\left[\theta_{k}+C_{b k m}-\bar{\phi}(\boldsymbol{\Theta})\right] \\
& -\sum_{r=1, r \neq k}^{N_{\mathrm{R}}} \sum_{b=1}^{N_{\mathrm{B}}} \sum_{m=1}^{N_{\mathrm{M}}} \frac{2}{N_{\mathrm{R}}}\left[\theta_{r}+C_{b r m}-\bar{\phi}(\boldsymbol{\Theta})\right]=0,
\end{aligned}
$$

for each $k \in \mathbb{K}$. After some manipulations, we get

$$
\begin{aligned}
& \left(1-\frac{1}{N_{\mathrm{R}}}\right) \theta_{k}-\frac{1}{N_{\mathrm{R}}} \sum_{r=1, r \neq k}^{N_{\mathrm{R}}} \theta_{r}+\frac{1}{N_{\mathrm{M}} N_{\mathrm{B}}} \\
& \times\left(\sum_{b=1}^{N_{\mathrm{B}}} \sum_{m=1}^{N_{\mathrm{M}}} C_{b k m}-\frac{1}{N_{\mathrm{R}}} \sum_{r=1}^{N_{\mathrm{R}}} \sum_{b=1}^{N_{\mathrm{B}}} \sum_{m=1}^{N_{\mathrm{M}}} C_{b r m}\right)=0 .
\end{aligned}
$$

Operating like this, the $N_{\mathrm{R}}$ linear equations in $N_{\mathrm{R}}$ unknowns (i.e., the RIS phases) can be simultaneously solved to obtain the phase shifts optimized for this specific problem. An optimal, albeit not unique, phase profile that minimizes the convexified objective function in (50) can be written in closedform as

$$
\hat{\theta}_{k}=\frac{2 \pi f_{\mathrm{c}}}{N_{\mathrm{M}} N_{\mathrm{B}}} \sum_{b=1}^{N_{\mathrm{B}}} \sum_{m=1}^{N_{\mathrm{M}}}\left[\tau_{b k}+\tau_{k m}-\frac{1}{N_{\mathrm{R}}} \sum_{r=1}^{N_{\mathrm{R}}}\left(\tau_{b r}+\tau_{r m}\right)\right] \text {. }
$$


Notably, the solution $\hat{\boldsymbol{\Theta}} \triangleq\left[\hat{\theta}_{1}, \hat{\theta}_{2}, \cdots, \hat{\theta}_{N_{\mathrm{R}}}\right]$ is not unique, since adding a constant phase shift $\phi_{c}$ to the RIS phases yields to the same value for the objective function in (50), as it will be accounted for also in the centroid $\bar{\phi}(\boldsymbol{\Theta})$. Indeed, even the objective function in (49) will not be changed by adding a constant phase shift because of the absolute operator. Having maximized the upper bound on the SNR, now we derive the constant phase $\phi_{c}$ such that the Cauchy-Schwarz inequality is tight, i.e, the phase difference between the direct and reflected links is minimal at the gNB; hence, the SNR is maximized. More precisely, substituting the optimal phases from (55) in (45) and maximizing it with respect to $\phi_{c}$ we get

$$
\underset{\phi_{c} \in[0,2 \pi)}{\operatorname{Maximize}} \frac{P}{\sigma^{2}}\left\|\mathbf{H}_{\mathrm{BM}} \mathbf{w}+e^{j \phi_{c}} \mathbf{H}_{\mathrm{BR}} \boldsymbol{\Omega}(\hat{\boldsymbol{\Theta}}) \mathbf{H}_{\mathrm{RM}} \mathbf{w}\right\|^{2} .
$$

Again, the number of degrees of freedom is not sufficient for adjusting the phase of the direct and reflected links for all the receiving antennas at the gNB. Hence, the Cauchy-Schwarz inequality can not be satisfied with equality, but can be made rather tight. In this regard, $\phi_{c}$ can be designed to minimize the difference between the phases of the direct and reflected links, i.e.,

$$
\begin{aligned}
\underset{\phi_{c} \in[0,2 \pi]}{\operatorname{Minimize}} & \gamma_{d}\left(\phi_{c}\right) \triangleq \sum_{r=1}^{N_{\mathrm{R}}} \sum_{b=1}^{N_{\mathrm{B}}} \sum_{m=1}^{N_{\mathrm{M}}}\left[\left(\beta_{m}-2 \pi f_{\mathrm{c}} \tau_{b m}\right)\right. \\
- & \left.\left(\phi_{c}+\hat{\theta}_{r}+\beta_{m}-2 \pi f_{\mathrm{c}}\left(\tau_{b r}+\tau_{r m}\right)\right)\right]^{2} .
\end{aligned}
$$

The optimal $\phi_{c}$ can be found by solving the following equation

$$
\begin{aligned}
& \frac{\partial \gamma_{d}\left(\phi_{c}\right)}{\partial \phi_{c}}=\sum_{r=1}^{N_{\mathrm{R}}} \sum_{b=1}^{N_{\mathrm{B}}} \sum_{m=1}^{N_{\mathrm{M}}}-2\left[-2 \pi f_{\mathrm{c}} \tau_{b m}+\beta_{m}\right. \\
& \left.-\left(\phi_{c}+\hat{\theta}_{r}+\beta_{m}-2 \pi f_{\mathrm{c}}\left(\tau_{b r}+\tau_{r m}\right)\right)\right]=0,
\end{aligned}
$$

leading to

$$
\hat{\phi}_{c}=\frac{2 \pi f_{\mathrm{c}}}{N_{\mathrm{R}} N_{\mathrm{M}} N_{\mathrm{B}}} \sum_{b=1}^{N_{\mathrm{B}}} \sum_{m=1}^{N_{\mathrm{M}}} \sum_{r=1}^{N_{\mathrm{R}}}\left(-\tau_{b m}+\tau_{b r}+\tau_{r m}\right),
$$

obtained by substituting the derived RIS phases in (55) and distribute the summations.

Finally, by combining (55) and (59), the designed phases for the RIS that accounts for the direct and reflected paths can be written, for each $k \in \mathbb{K}$, as

$$
\theta_{k}^{*}=\hat{\theta}_{k}+\hat{\phi}_{c}=\frac{2 \pi f_{c}}{N_{\mathrm{M}} N_{\mathrm{B}}} \sum_{b=1}^{N_{\mathrm{B}}} \sum_{m=1}^{N_{\mathrm{M}}}\left(\tau_{b k}+\tau_{k m}-\tau_{b m}\right) .
$$

Since the proposed RIS phases do not depend on the beamforming vector, we can select any appropriate beamformer that maximizes the SNR, e.g., eigen-beamforming, albeit it is not always optimal from the positioning and orientation detection perspective. Note also that the previous analysis considers $\tau_{k m}$ and $\tau_{b m}$ to be a-priori known to provide the fundamental attainable limits. In practice, sub-optimal phases can be obtained by substituting the channel parameters, e.g., the TOAs and bearing angles, in (43), (44), or (60) with their estimates rather than their true values [76], [77]. For instance, a localization algorithm can start with a random RIS phase design; then, obtain an estimate for the TOAs, update the phase design, and repeat these steps till convergence [78].

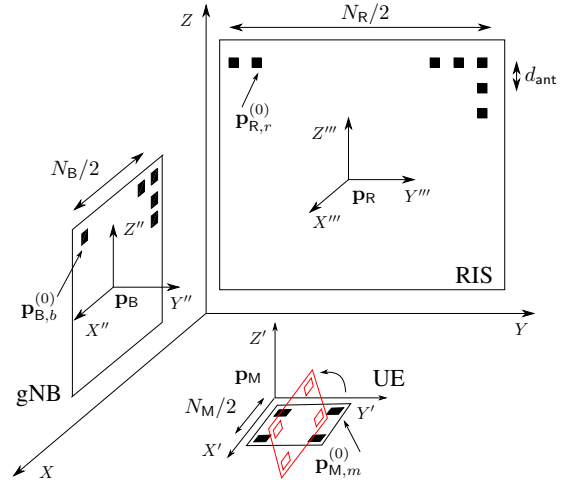

Fig. 3: Considered 3D localization scenario. An example of rotated UE is depicted in red.

\section{Numerical RESUlts}

\section{A. Simulation Parameters}

According to the previous analysis, we now evaluate the attainable localization and orientation performance limits for different scenarios. More specifically, we here focus on planar antenna array configuration, ${ }^{3}$ as they allow compact deployment of massive arrays in gNBs and UE, as well as 3D beamfocusing capabilities [79], [80]. Moreover, in the perspective to place RIS on walls, planar geometry represents a practical solution [1], [36].

The $\mathrm{gNB}$ is assumed to be located at the origin, i.e., at $\mathbf{p}_{\mathrm{B}} \triangleq$ $\left[x_{\mathrm{B}}, y_{\mathrm{B}}, z_{\mathrm{B}}\right]^{\top}=(0,0,0)(\mathrm{m})$, if not otherwise indicated and the initial positions of the antennas (in absence of rotations) can be represented as in Fig. 3 where the gNB, RIS, and UE are lying on the $X Z$ - and $Y Z$-, $X Y$ - planes, respectively, and the coordinates of the array elements are given by

$\mathbf{p}_{\mathrm{B}, i}^{(0)}=d_{\mathrm{ant}}\left[\left\lfloor\frac{i}{\sqrt{N_{\mathrm{B}}}}\right\rfloor, 0,\left(i \bmod \sqrt{N_{\mathrm{B}}}\right)\right]^{\top}, i \in\left\{1, \ldots, N_{\mathrm{B}}\right\}$,
$\mathbf{p}_{\mathrm{R}, i}^{(0)}=d_{\mathrm{ant}}\left[0,\left\lfloor\frac{i}{\sqrt{N_{\mathrm{R}}}}\right\rfloor,\left(i \bmod \sqrt{N_{\mathrm{R}}}\right)\right]^{\top}, i \in\left\{1, \ldots, N_{\mathrm{R}}\right\}$,
$\mathbf{p}_{\mathrm{M}, i}^{(0)}=d_{\mathrm{ant}}\left[\left\lfloor\frac{i}{\sqrt{N_{\mathrm{M}}}}\right\rfloor,\left(i \bmod \sqrt{N_{\mathrm{M}}}\right), 0\right]^{\top}, i \in\left\{1, \ldots, N_{\mathrm{M}}\right\}$,

where $\bmod$ is the modulo operator, $d_{\mathrm{ant}}=\lambda / 2$ is the interantenna spacing, and the rotated antenna elements for a given roll, pitch and yaw angles can be defined as in (7). In particular, while the gNB and the RIS are fixed on the $X Z$ - and $Y Z$ - planes (i.e., $\alpha_{\mathrm{B}}=\beta_{\mathrm{B}}=\gamma_{\mathrm{B}}=0, \alpha_{\mathrm{R}}=\beta_{\mathrm{R}}=\gamma_{\mathrm{R}}=0$ ), respectively, the UE can freely rotate around $x-, y-$, and $z-$ axis with angles $\gamma_{\mathrm{M}}, \beta_{\mathrm{M}}$, and $\alpha_{\mathrm{M}}$, respectively, according to Fig. 2.

At the transmitter, we considered OFDM signalling with $N=12$ subcarriers, transmitted power per subcarrier $P=$ $-10 \mathrm{dBm}$, subcarrier spacing $\Delta f=240 \mathrm{kHz}$, and carrier frequency $f_{\mathrm{c}}=28 \mathrm{GHz}$ [81]. At the receiver, the noise variance per subcarrier is set as $\sigma^{2}=K \mathrm{~F} T_{0} \Delta f=-117.17 \mathrm{dBm}$,

\footnotetext{
${ }^{3}$ Note that the previous analysis is valid for any geometric configuration, i.e., any spatial deployment and orientation for the antenna arrays.
} 


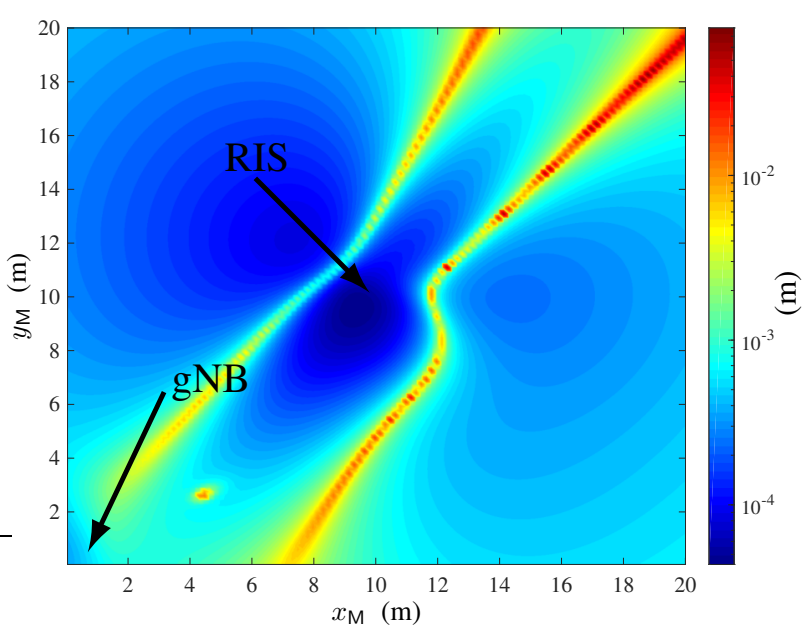

(a) PEB

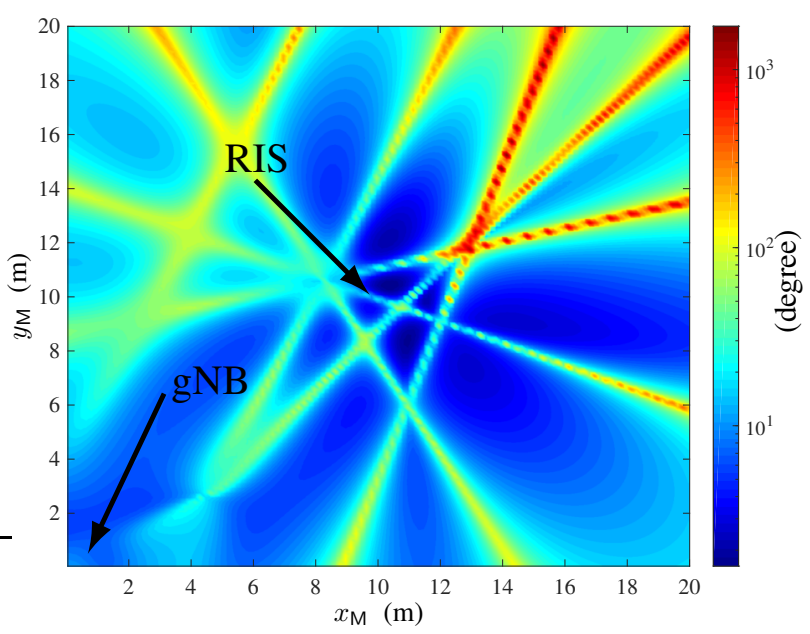

(b) OEB

Fig. 4: (a) PEB in meters and (b) OEB in degrees for different mobile locations in an area of $20 \times 20 \mathrm{~m}^{2}$ on the $X Y$-plane. The orientation of the UE is set to $\phi_{\mathrm{M}}=(\pi / 6, \pi / 6, \pi / 6)$.

where $K$ is the Boltzmann constant, $T_{0}=290 \mathrm{~K}$ is the receiver temperature and $\mathrm{F}=3 \mathrm{~dB}$ is the noise figure.

Concerning the RIS phase profile in (9), in the next, we use the following labels according to the type of design: $(i)$ Mirror, when the RIS does not induce any phase shift, that is $\boldsymbol{\Theta}=\mathbf{0}_{1 \times N_{\mathrm{R}}}$; (ii) Random, when the RIS phase shifts are uniformly distributed between 0 and $2 \pi ;^{4}$ (iii) SNR-based, according to the analysis of Sec. IV-2 maximizing the SNR; (iv) Optimized CRLB-based, according to the minimization of the CRLB reported in Sec. IV-1, and; (v) Quantized, that accounts for 4 quantization levels in the representation of the optimized CRLB. We consider the beamforming vector as $\mathbf{w}=[1,1, \cdots, 1]^{\top} / \sqrt{N_{\mathrm{M}}}$ in all the next numerical results, except for Table I, where we also account for EigenBeamforming and a numerically optimized beamformer.

\footnotetext{
${ }^{4}$ For obtaining this result, we simulated 100 random phase configuration and average the theoretical PEB/OEB over them.
}

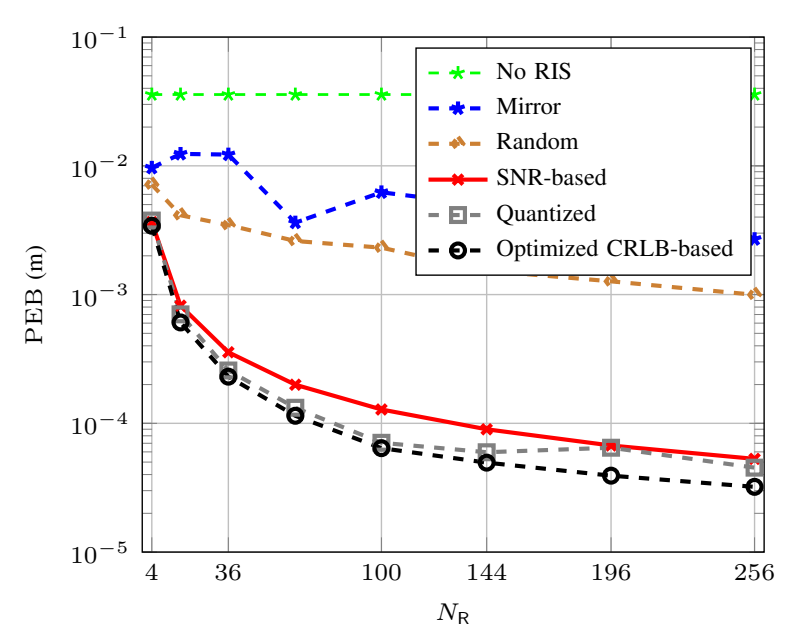

(a) PEB

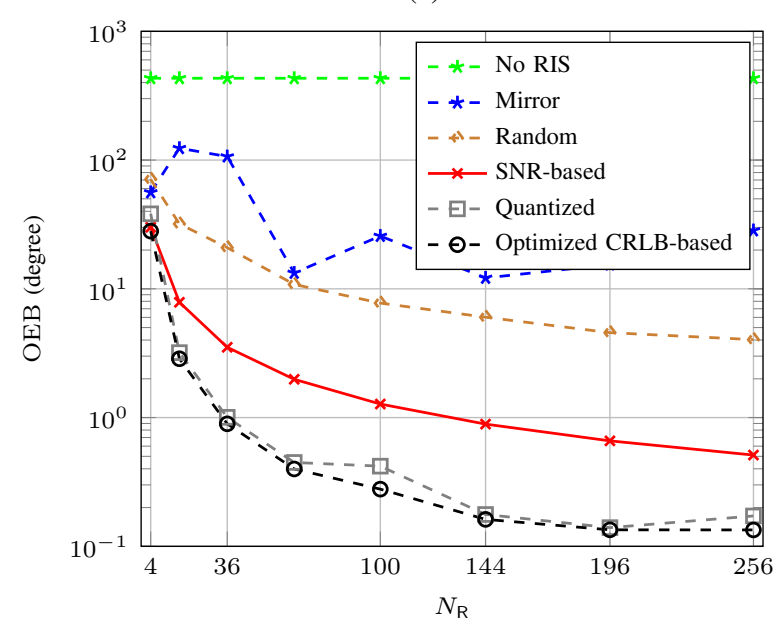

(b) OEB

Fig. 5: PEB and OEB vs. number of RIS elements, namely $N_{\mathrm{R}}$, for different RIS phase design strategies.

\section{B. Numerical Results}

a) PEB and OEB for Different Mobile Positions: Fig. 4 shows the position and orientation errors in RIS-assisted architecture by varying the UE location in different points of the area. In particular, the location of the RIS is $\mathbf{p}_{\mathrm{R}} \triangleq$ $\left[x_{\mathrm{R}}, y_{\mathrm{R}}, z_{\mathrm{R}}\right]^{\top}=(10,10,-1)(\mathrm{m})$, whereas the $\mathrm{gNB}$ is placed in $\mathbf{p}_{\mathrm{B}}=(0,0,0)$, if not otherwise indicated. The $\mathrm{gNB}$, RIS, and UE are equipped with planar antenna arrays with $N_{\mathrm{B}}=36, N_{\mathrm{R}}=100, N_{\mathrm{M}}=4$ antennas, respectively.

The UE altitude is set to $z_{\mathrm{M}}=-3 \mathrm{~m}$, the UE orientation to $\phi_{\mathrm{M}} \triangleq\left[\alpha_{\mathrm{M}}, \beta_{\mathrm{M}}, \gamma_{\mathrm{M}}\right]^{\top}=(\pi / 6, \pi / 6, \pi / 6)$ (rad), and the proposed SNR-based phase design is adopted for the RIS.

As it can be seen in Fig. $4 a$ and Fig. 4b, the PEB and the OEB are lower in proximity of the gNB and of the RIS, with an error of about $8 \times 10^{-4} \mathrm{~m}$ for the position and of $10.7^{\circ}$ for the orientation when the UE is placed at $\mathbf{p}_{\mathrm{M}} \triangleq\left[x_{\mathrm{M}}, y_{\mathrm{M}}, z_{\mathrm{M}}\right]^{\top}=$ $(4,4,-3)(\mathrm{m})$. Notably, the achieved errors depend not only on the distance from the gNB and from the RIS, but also on the relative UE location with respect to them, e.g., the UE location has an effect on the actual bearing angles $\phi_{\mathrm{RM}}$ and 


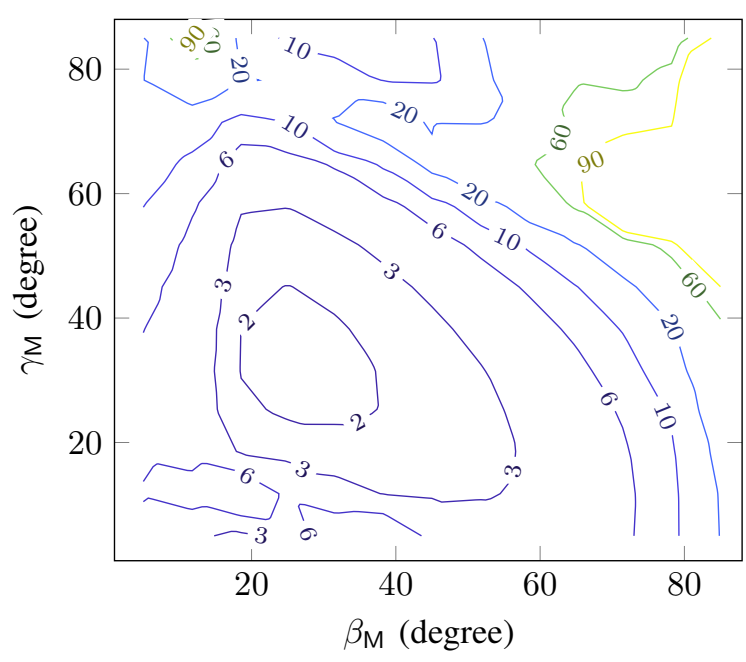

Fig. 6: OEB contours as a function of the orientation angles $\beta_{\mathrm{M}}$ and $\gamma_{\mathrm{M}}$ in degrees for a fixed $\alpha_{\mathrm{M}}=30^{\circ}$.

$\phi_{\mathrm{BM}}$ and, in turns, on the localization.

b) PEB and OEB for Different RIS Configurations:

Fig. 5 reports the localization and orientation error bounds, derived in (28), for different number of antennas at the RIS and different phase design strategies. We set the number of gNB antennas to $N_{\mathrm{B}}=16$, the number of UE antennas to $N_{\mathrm{M}}=4$, the RIS and UE centroids to $\mathbf{p}_{\mathrm{R}}=(4,3,1)$ (m) and $\mathbf{p}_{\mathrm{M}}=(5,2,-1)(\mathrm{m})$, respectively. The UE orientation is set to $\phi_{\mathrm{M}}=(\pi / 6, \pi / 6, \pi / 6)$ (rad).

As previously discussed, the Optimized CRLB phase design strategy is obtained by numerically minimizing the PEB and OEB. Because the CRLB optimization problem is non-convex, the algorithm could converge to a local minimum if the initial point is far from the true solution. Therefore, we included the proposed closed-form phase design in (60) as a possible initialization for the optimization algorithm in the Optimized $C R L B$ phase design. Moreover, for each Monte Carlo iteration, we have generated a different Random phase profile for the RIS. For the PEB in Fig. 5a, we can see that the proposed $S N R$-based design almost coincides with the Optimized CRLB, and that the quantization does not significantly decrease the performance. This is also due to the fact that the CauchySchwartz inequality is satisfied with almost equality with the proposed RIS phase design, as verified numerically.

Regarding the OEB in Fig. 5b, the optimized CRLB-based and its quantized version allow to outperform the proposed SNR-based scheme. Another interesting aspect is that the error tends to slowly decrease for $N_{\mathrm{R}} \geq 100$, thus permitting to relax the number of antennas at the RIS side while obtaining the good localization performance. Also, all RIS-assisted scenarios outperform the No RIS case, regardless of the phase design.

c) Analysis of the UE Orientation: We now analyze the impact of the mobile orientation angle on the OEB when the location of the mobile and its orientation with respect to the $z$-axis are fixed, i.e., $\mathbf{p}_{\mathrm{M}}=(15,5,-3)(\mathrm{m})$ and $\alpha_{\mathrm{M}}=\pi / 6$, respectively, while the orientation of the UE around both $x$ and $y$ - axis (i.e., $\beta_{\mathrm{M}}$ and $\gamma_{\mathrm{M}}$ ) are varied from 0 to $\pi / 2$. The

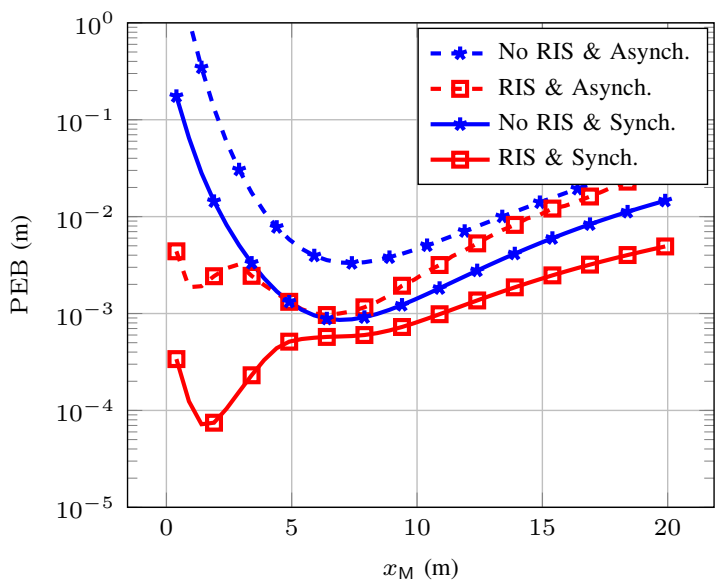

(a) $\mathbf{p}_{\mathrm{M}}=\left(x_{\mathrm{M}}, 5,1\right)$

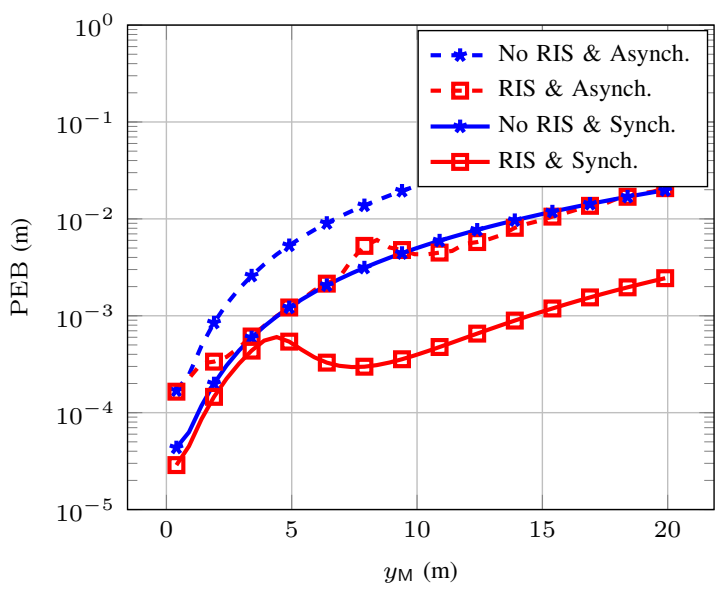

(b) $\mathbf{p}_{\mathrm{M}}=\left(5, y_{\mathrm{M}}, 1\right)$

Fig. 7: PEB for synchronous and asynchronous signal models, for UE orientation fixed to $\phi_{\mathrm{M}}=(\pi / 4, \pi / 2,0)$ for $\mathbf{p}_{\mathrm{B}}=$ $(5,0,1.5)$ and $\mathbf{p}_{\mathrm{R}}=(0,5,2)$.

number of antennas at the gNB, UE and RIS are $N_{\mathrm{B}}=36$, $N_{\mathrm{M}}=4$, and $N_{\mathrm{R}}=256$, respectively. The RIS position is $\mathbf{p}_{\mathbf{R}}=(10,10,-1)(\mathrm{m})$. In this sense, according to the results reported in Fig.6, we can observe that the OEB increases when the mobile is parallel and/or perpendicular to the RIS and the gNB. On the other hand, the OEB decreases when $\gamma_{M}$ and $\beta_{M}$ are close to $30^{\circ}$.

d) Synchronous vs. Asynchronous signalling: We now compare the achievable performance of synchronous and asynchronous systems in an environment with and without RIS. More specifically, we evaluated the PEB as a function of the $x / y$-coordinates of the mobile for a fixed UE orientation, i.e., $\phi_{\mathrm{M}}=(\pi / 4, \pi / 2,0)$ (see Fig. 7), and for averaged orientations, i.e., for 36 configurations where both $\alpha_{\mathrm{M}}$ and $\beta_{\mathrm{M}}$ are varied between $0^{\circ}$ and $90^{\circ}$ degrees with a step of $15^{\circ}$ degree (see Fig. 8). In both configurations, the gNB and the RIS are located at $\mathbf{p}_{\mathrm{B}}=(5,0,1.5)$ and $\mathbf{p}_{\mathrm{R}}=(0,5,2)$, respectively. The number of antennas are $N_{\mathrm{B}}=36, N_{\mathrm{R}}=64$, and $N_{\mathrm{M}}=4$. In particular, Fig. 7-(a) and Fig. 8-(a) are obtained by fixing $y_{\mathrm{M}}=5 \mathrm{~m}$, with $x_{\mathrm{M}}$ spanning from 0 to $20 \mathrm{~m}$, whereas in Fig. 7-(b) and in Fig. 8-(b) we set $x_{\mathrm{M}}=5 \mathrm{~m}$ and $y_{\mathrm{M}}$ is changed 


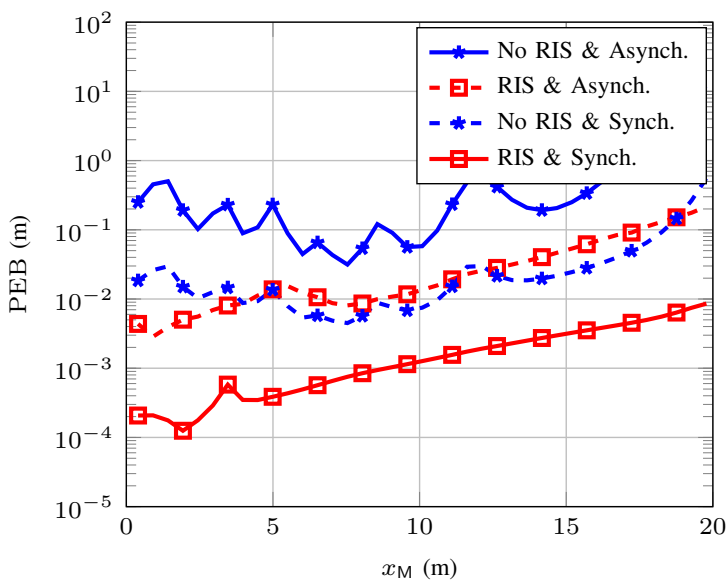

(a) $\mathbf{p}_{\mathrm{M}}=\left(x_{\mathrm{M}}, 5,1\right)$

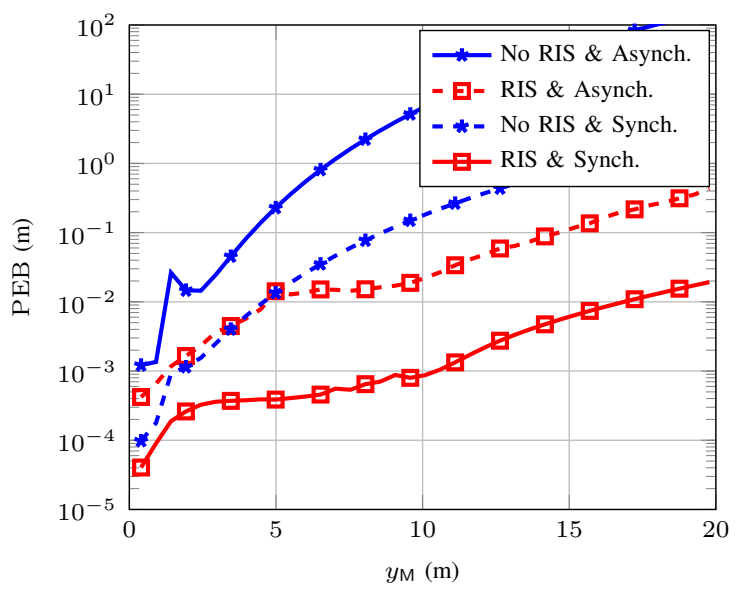

(b) $\mathbf{p}_{\mathrm{M}}=\left(5, y_{\mathrm{M}}, 1\right)$

Fig. 8: PEB for synchronous and asynchronous signal models, averaged for different UE orientation for $\mathbf{p}_{\mathrm{B}}=(5,0,1.5)$ and $\mathbf{p}_{\mathbf{R}}=(0,5,2)$.

from 0 to $20 \mathrm{~m}$. We can see that the PEB performance, for the synchronous case always outperforms the asynchronous one (even of few order of magnitudes), thanks to the availability of a larger set of information. Moreover, the PEB is strongly affected by the geometry, and it generally keeps the lowest values in the area between the gNB and the RIS.

The orientation of the mobile impacts the localization performance, as it can be seen in Fig. 8. Hence, we averaged over several mobile orientations to study the effect of the distance on the localization, regardless of the mobile orientation. As expected, when increasing the UE distance from the gNB, e.g., by varying the $y$-coordinate, the localization error increases faster to what happens by moving along the $x$-axis (i.e., far from the RIS). Note also that, when the UE moves far from the gNB, the geometry is such that the contribution of the RIS is more beneficial for positioning.

e) Two-stage Localization: In Fig. 9, the localization accuracy is investigated for the case that the system can estimate only a subset of the parameters in (26).

In particular, we differentiate between two cases: $(i)$ the RSSI and AOA are estimated; (ii) the TOAs and the AOA

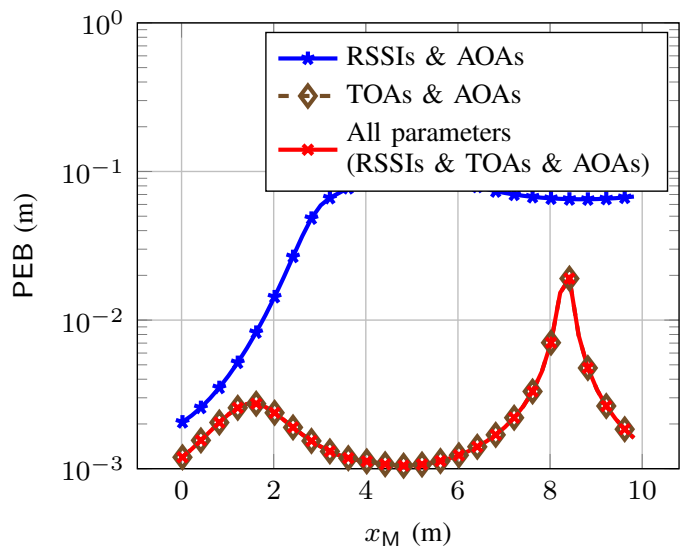

Fig. 9: PEB for different $x$-coordinates of a UE located in $\mathbf{p}_{\mathrm{M}}=\left(x_{\mathrm{M}}, 2,-3\right)$. The RIS is located at $\mathbf{p}_{\mathrm{R}}=(4,4,-1)$.

are estimated. Then, the two scenarios are compared with the benchmark, where the system can estimate all the parameters in (26), and the corresponding PEB is calculated as in (28). The PEB for the three cases is depicted in Fig. 9 for various values of mobile locations, along the $x$-axis. In the considered scenario, we set $\mathbf{p}_{\mathrm{M}}=\left(x_{\mathrm{M}}, 2,-3\right), \mathbf{p}_{\mathrm{R}}=(4,4,-1)$, $N_{\mathrm{B}}=16, N_{\mathrm{R}}=36$, and $N_{\mathrm{M}}=4$. We can see that discarding the RSSIs from the parameter vector (i.e., not relying on measuring the RSSIs for positioning purposes) has negligible impact on the PEB. On the contrary, if the system is able only to estimate the RSSI and not the TOAs, the localization error increases up to two orders of magnitude. Therefore, in our considered setting, localization systems with accurate TOA estimation can achieve higher performance compared to those relying on RSSIs.

For the two-stage approach in estimating the location, it is beneficial to quantify the minimum possible error for estimating the parameters in (26). To this purpose, the error bound on the parameters can be written as

$$
\sigma_{\Gamma_{j}} \triangleq \sqrt{\left[\sum_{n=1}^{N} \mathbf{I}_{n}(\boldsymbol{\Gamma})\right]_{j, j}^{-1}}, \quad \forall j \in\{1,2, \cdots,|\boldsymbol{\Gamma}|\},
$$

where $\mathbf{I}_{n}(\boldsymbol{\Gamma})$ is the FIM of the parameters for a given subcarrier $n$ as expressed in (30) and $|\boldsymbol{\Gamma}|$ is the number of parameters in $\boldsymbol{\Gamma}$. In this regard, we depict in Fig. 10 the error for estimating the parameters $\theta_{\mathrm{BM}}, \phi_{\mathrm{BM}}, \tau_{\mathrm{BM}}, \theta_{\mathrm{RM}}, \phi_{\mathrm{RM}}, \tau_{\mathrm{RM}}$. In particular, the errors in estimating the time, i.e., $\sigma_{\tau_{\mathrm{BM}}}$ and $\sigma_{\tau_{\mathrm{RM}}}$, are shown in Fig. 10a, while the standard deviation of the estimation errors of the angles, i.e., $\sigma_{\theta_{\mathrm{BM}}}, \sigma_{\phi_{\mathrm{BM}}}$ and $\sigma_{\theta_{\mathrm{RM}}}$, $\sigma_{\phi_{\mathrm{RM}}}$, are presented in Fig. 10b. We can see that the parameters that depend on the gNB, i.e., $\sigma_{\tau_{\mathrm{BM}}}, \sigma_{\theta_{\mathrm{BM}}}, \sigma_{\phi_{\mathrm{BM}}}$, have minimum estimation errors near the gNB location. On the other hand, the parameters where the RIS is involved increase as UE gets far from the RIS.

f) Impact of the Beamforming Technique: In Table I, we compare the PEB performance for various transmit beamforming techniques along with several RIS phase designs using AO. In particular, we consider three possible beamforming techniques: (i) No Beamforming, with $\mathbf{w}=[1,1, . ., 1] / \sqrt{N_{\mathrm{M}}}$; (ii) 


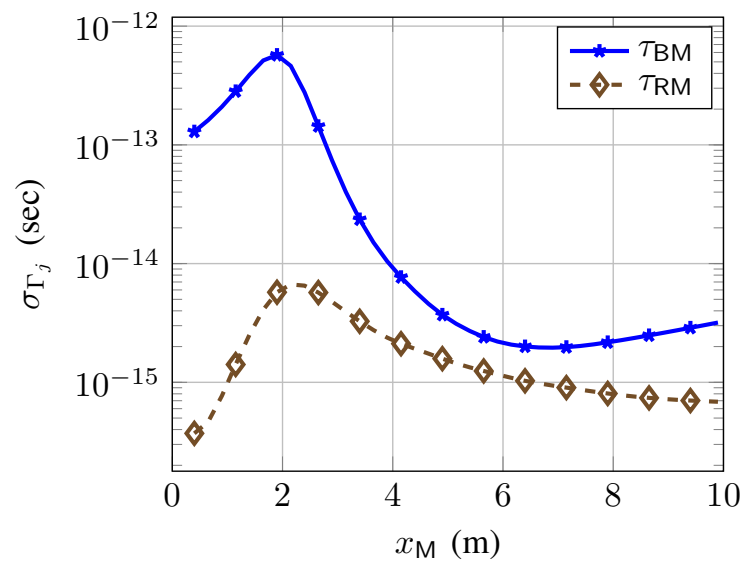

(a) Error bounds on the TOA estimation

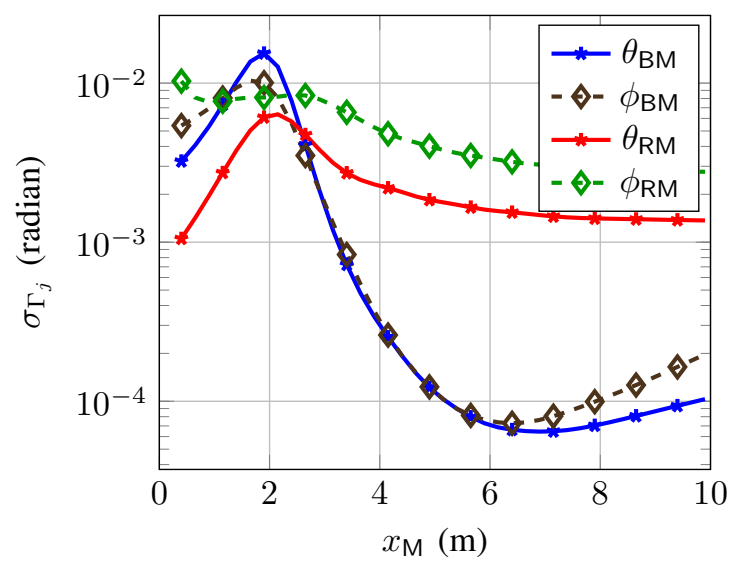

(b) Error bounds on the angles estimation

Fig. 10: The error in estimating the quantities in the parameters vector vs the $x$-coordinates of the mobile location, for $\mathbf{p}_{\mathrm{B}}=(5,0,1.5), \mathbf{p}_{\mathrm{R}}=(0,5,2), \phi_{\mathrm{M}}=(\pi / 4, \pi / 2,0)$, $\mathbf{p}_{\mathrm{M}}=\left(x_{\mathrm{M}}, 3,1\right), N_{\mathrm{B}}=36, N_{\mathrm{R}}=64$, and $N_{\mathrm{M}}=4$.

Eigen-Beamforming, with the power-normalized eigenvector (corresponding to the maximum eigenvalue) of $\mathbf{H}^{\mathrm{H}} \mathbf{H}$, with $\mathbf{H} \triangleq \mathbf{H}_{\mathrm{BM}}+\mathbf{H}_{\mathrm{BR}} \boldsymbol{\Omega}(\boldsymbol{\Theta}) \mathbf{H}_{\mathrm{RM}}$ being the effective channel between the UE and the gNB [82], computed with $f_{n}=f_{c}$; (iii) Optimized, with numerically optimized beamformer to minimize the PEB for a fixed RIS phase profile. Then, we set $N_{\mathrm{B}}=16, N_{\mathrm{M}}=4, N_{\mathrm{R}}=36, \mathbf{p}_{\mathrm{R}}=(5,5,1)$, and $\mathbf{p}_{\mathrm{M}}=(10,3,-1)$. We can see from Table I that optimizing both the beamformer and the RIS phases through AO achieves a significant gain, almost two orders of magnitude compared to schemes where beamforming and/or RIS phases are not optimized. A similar performance can be achieved for Eigenbeamforming. Note that the proposed SNR-based RIS phase design when combined with Eigen-Beamforming gives unsatisfactory results. This behavior can be attributed to the fact that both schemes aim to maximize the SNR by aligning the phases at the receiving antennas, regardless the location of the users. Hence, we lost some degrees of diversity on the information about the user locations resulting in higher errors.
TABLE I: The PEB $(\mathrm{cm})$ for various RIS phase and beamforming designs.

\begin{tabular}{|l|c|c|c|}
\hline RIS Phase Beamformer & No & Eigen-Beamformer & Optimized CRLB-based \\
\hline Mirror & 0.615 & 0.25 & 0.20 \\
\hline SNR-based & 0.077 & 0.738 & 0.037 \\
\hline Optimized CRLB-based & 0.023 & 0.006 & 0.006 \\
\hline
\end{tabular}

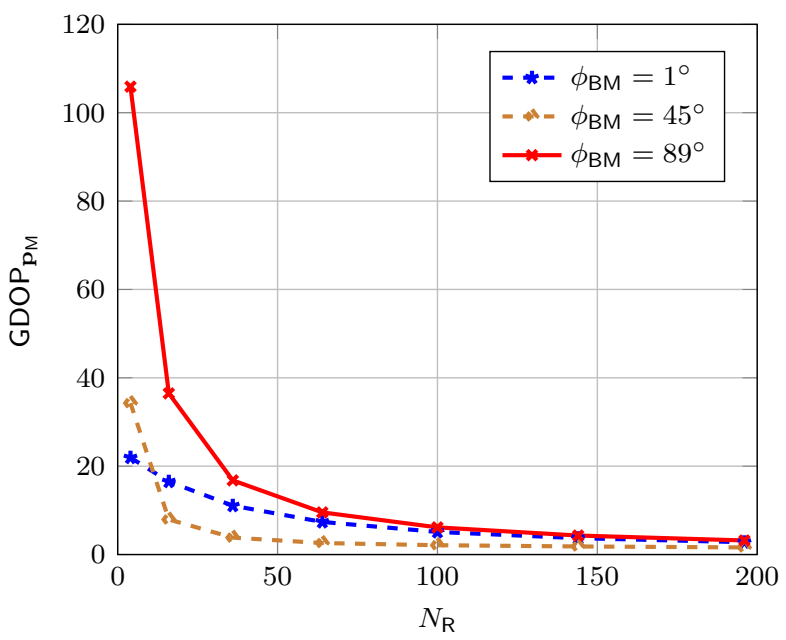

(a) The GDOP for the mobile position.

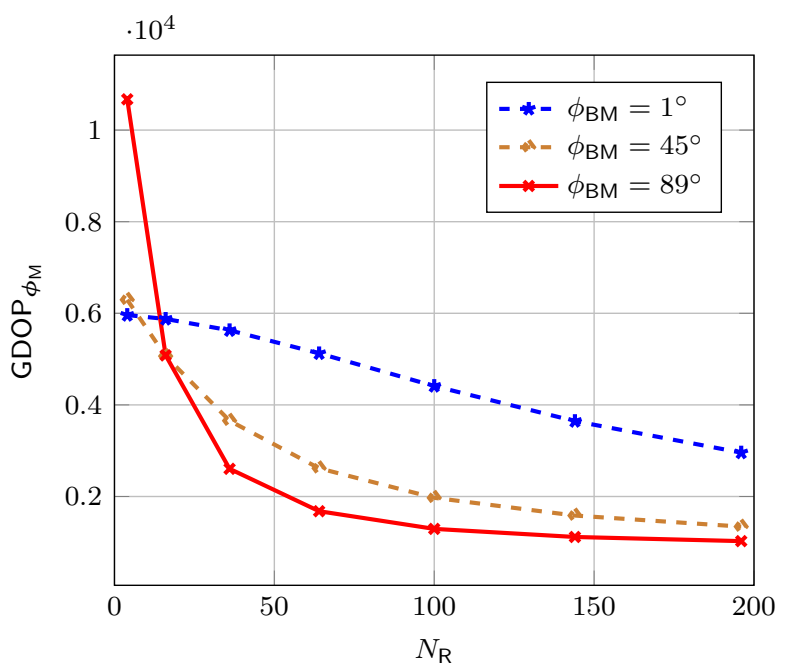

(b) The GDOP for the orientation angles.

Fig. 11: The GDOP for various $N_{\mathrm{R}}$ and $\phi_{\mathrm{BM}}$, for $N_{\mathrm{B}}=36$, $N_{\mathrm{M}}=16, \mathbf{p}_{\mathrm{R}}=(4,3,1), d_{\mathrm{BM}}=3 \mathrm{~m}, \alpha_{\mathrm{M}}=30$, and $N=2400$.

g) Geometric dilution of precision: In Fig. 11, the impact of the geometry on the localization and orientation estimation errors is investigated as a function of the number of elements in the RIS. The GDOP value can be considered as an amplification of the estimation error due to the geometry. Therefore, smaller values of the GDOP indicate a favorable geometry of the mobile with respect to both the gNB and the RIS. The GDOP for the position, GDOP ${ }_{\mathbf{p}_{M}}$, is depicted in Fig. 11a as a function of the number of RIS elements for various UE locations with different azimuth angles between the gNB and $\mathrm{UE}, \phi_{\mathrm{BM}}$, while the corresponding distance and the elevation 
angle are fixed to $d_{\mathrm{BM}}=3 \mathrm{~m}$ and $\theta_{\mathrm{BM}}=30^{\circ}$, respectively. It can be noticed that increasing the number of RIS elements tends to enhance the geometry of the problem and, thus, it can reduce the positioning error. Also, the GDOP depends strongly on the azimuth angle for small $N_{\mathrm{R}}$ and, consequently, on the UE orientation. The same behavior can be seen in Fig. $11 \mathrm{~b}$ for the GDOP related to the orientation, i.e., GDOP $\phi_{\mathrm{M}}$. The main difference is that it is more harder to estimate the orientation angle with a small number of RIS elements compared to the position estimation. In fact, the GDOP can be interpreted as a mapping from the standard deviation of the thermal noise to the estimation error in terms of the PEB and OEB. For example, with $N_{\mathrm{R}}=36$ and $\phi_{\mathrm{BM}}=45^{\circ}$, we have $\mathrm{PEB} \approx 3.8\left(d_{\mathrm{BM}} / \sqrt{P}\right) \sigma$, while OEB $\approx(3650 / \sqrt{P}) \sigma$, from (41) and (42). We can see that, for a fixed number of RIS elements, the position can be estimated with a higher precision with respect to the orientation. Also, the GDOP for the orientation decreases for RISs with larger number of elements. The reason is that the orientation estimation relies on the curvature of the wavefront in the near-field region. Hence, for a larger number of elements, the effective size of the RIS increases along with the Fraunhofer distance [62].

\section{CONCLUSIONS}

In this paper, we proposed an architecture for joint communication and UE localization and orientation estimation in a RIS-assisted environment. We derived the ultimate performance in terms of PEB and OEB, accounting for both near- and far-field propagation conditions. The RIS phases were designed to maximize the SNR towards the desired UE for both communication and localization enhancement. Indeed, we obtained that the RIS with the proposed SNRbased phase design can significantly increase the localization performance by focusing the incident spherical wavefront from the UE toward the gNB. The proposed SNR-based scheme, when compared to a conventional system without RIS, can achieve up to two orders and one order of magnitude reduction in PEB and OEB, respectively. Also, the localization accuracy strongly depends on the considered geometry and the orientation of the UE. The achieved results open the door towards the adoption of RISs as an effective mean for supporting mobile wireless localization and, thus, boosting the communication performance. A step forward will be the analysis of the localization performance limits in multi-RISs scenarios, or the design of ad-hoc localization algorithms in the presence of multi-scatterers, accounting for proper channel models [83]. In this sense, the provided bounds can serve as a benchmark for comparing the attainable performance with the new algorithms.

\section{ACKNOWLEDGEMENTS}

Authors wish to thank D. Dardari for motivating this work and A. Abdelhady for the useful discussions.

\section{APPENDIX A}

THE JACOBIAN MATRIX

In this appendix, we report the elements of the Jacobian matrix for the CRLB derivation in (27). The Jacobian matrix of the mobile location is given by (63) in the top of next page where for each $a_{\mathrm{M}} \in\left\{x_{\mathrm{M}}, y_{\mathrm{M}}, z_{\mathrm{M}}\right\}$ and $\mathrm{S} \in\{\mathrm{B}, \mathrm{R}\}$, the following relationships hold

$$
\begin{aligned}
& \nabla_{a_{\mathrm{M}}} \tau_{\mathrm{SM}}=\frac{\nabla_{a_{\mathrm{M}}} d_{\mathrm{SM}}}{c}=\frac{1}{c} \frac{a_{\mathrm{M}}-a_{\mathrm{S}}}{d_{\mathrm{SM}}}, \\
& \nabla_{a_{\mathrm{M}}} \phi_{\mathrm{SM}}=\frac{1}{1+\left(\frac{y_{\mathrm{M}}-y_{\mathrm{S}}}{x_{\mathrm{M}}-x_{\mathrm{S}}}\right)^{2}} \nabla_{a_{\mathrm{M}}}\left(\frac{y_{\mathrm{M}}-y_{\mathrm{S}}}{x_{\mathrm{M}}-x_{\mathrm{S}}}\right), \\
& \nabla_{a_{\mathrm{M}}} \theta_{\mathrm{SM}}=\frac{1}{\sqrt{1-\left(\frac{z_{\mathrm{M}}-z_{\mathrm{S}}}{d_{\mathrm{SM}}}\right)^{2}}} \nabla_{a_{\mathrm{M}}}\left(\frac{z_{\mathrm{M}}-z_{\mathrm{S}}}{d_{\mathrm{SM}}}\right), \\
& \nabla_{a_{\mathrm{M}}} \rho_{\mathrm{BM}}=-\frac{\lambda}{4 \pi} \frac{1}{\left(d_{\mathrm{BM}}\right)^{2}} \frac{a_{\mathrm{M}}-a_{\mathrm{B}}}{d_{\mathrm{BM}}}, \\
& \nabla_{a_{\mathrm{M}}} \rho_{\mathrm{BRM}}=-\frac{\lambda}{4 \pi} \frac{1}{\left(d_{\mathrm{RM}}+d_{\mathrm{BR}}\right)^{2}} \frac{a_{\mathrm{M}}-a_{\mathrm{R}}}{d_{\mathrm{RM}}} .
\end{aligned}
$$

After some manipulation, (65)-(66) can be simplified as

$$
\left\{\begin{array} { l } 
{ \nabla _ { x _ { \mathrm { M } } } \phi _ { \mathrm { SM } } = - \frac { 1 } { d _ { \mathrm { SM } } } \frac { \operatorname { s i n } \phi _ { \mathrm { SM } } } { \operatorname { c o s } \theta _ { \mathrm { SM } } } } \\
{ \nabla _ { y _ { \mathrm { M } } } \phi _ { \mathrm { SM } } = \frac { 1 } { d _ { \mathrm { SM } } } \frac { \operatorname { c o s } \phi _ { \mathrm { SM } } } { \operatorname { c o s } \theta _ { \mathrm { SM } } } } \\
{ \nabla _ { z _ { \mathrm { M } } } \phi _ { \mathrm { SM } } = 0 }
\end{array} \quad \left\{\begin{array}{l}
\nabla_{x_{\mathrm{M}}} \theta_{\mathrm{SM}}=-\frac{\sin \left(\theta_{\mathrm{SM}}\right) \cos \left(\phi_{\mathrm{SM}}\right)}{d_{\mathrm{SM}}} \\
\nabla_{y_{\mathrm{M}}} \theta_{\mathrm{SM}}=-\frac{\sin \left(\theta_{\mathrm{SM}}\right) \sin \left(\phi_{\mathrm{SM}}\right)}{d_{\mathrm{SM}}} \\
\nabla_{z_{\mathrm{M}}} \theta_{\mathrm{SM}}=\frac{\cos \left(\theta_{\mathrm{SM}}\right)}{d_{\mathrm{SM}}} .
\end{array}\right.\right.
$$

\section{APPENDIX B \\ FIM ELEMENTS}

In order to derive the elements of the FIM in (34), the derivatives of the mean received signal with respect to the parameters, i.e., $\partial \mu_{b}[n] / \partial \Gamma_{j}$, should be derived for each $\Gamma_{j} \in$ $\boldsymbol{\Gamma}$. Let us first rewrite (12) as

$$
\mu_{b}[n]=\mu_{b, \mathrm{BM}}[n]+\mu_{b, \mathrm{BRM}}[n],
$$

with

$$
\begin{aligned}
& \mu_{b, \mathrm{BM}}[n] \triangleq \sqrt{P} \rho_{\mathrm{BM}} \sum_{m=1}^{N_{\mathrm{M}}} \mu_{b m}[n], \\
& \mu_{b, \mathrm{BRM}}[n] \triangleq \sqrt{P} \rho_{\mathrm{BRM}} \sum_{m=1}^{N_{\mathrm{M}}} \sum_{r=1}^{N_{\mathrm{R}}} \mu_{b r m}[n] .
\end{aligned}
$$

The signal inside the summation is

$$
\begin{aligned}
& \mu_{b m}[n] \triangleq x_{m}[n] \exp \left(-j 2 \pi f_{n}\left(\tilde{\tau}_{b m}+\tilde{\xi}_{\mathrm{BM}}+\eta_{m}\right)\right), \\
& \mu_{b r m}[n] \triangleq x_{m}[n] \exp \left(j \theta_{r}\right) \\
& \times \exp \left(-j 2 \pi f_{n}\left(\tilde{\tau}_{b r}+\tilde{\tau}_{r m}+\eta_{r}+\tilde{\xi}_{\mathrm{BRM}}+\eta_{m}\right)\right),
\end{aligned}
$$

where we have the following definitions for the synchronous signalling: $\tilde{\xi}_{\mathrm{BM}} \triangleq \xi_{\mathrm{BM}}, \tilde{\xi}_{\mathrm{BRM}} \triangleq \xi_{\mathrm{BM}}, \tilde{\tau}_{b m} \triangleq \tau_{b m}, \tilde{\tau}_{r m} \triangleq \tau_{r m}$, and $\tilde{\tau}_{b r} \triangleq \tau_{b r}$; whereas for the asynchronous signalling it is: $\tilde{\xi}_{\mathrm{BM}} \triangleq \chi_{\mathrm{BM}} / 2 \pi f_{n}, \tilde{\xi}_{\mathrm{BRM}} \triangleq \chi_{\mathrm{BRM}} / 2 \pi f_{n}, \tilde{\tau}_{b m} \triangleq \Delta \tau_{b m}$, $\tilde{\tau}_{r m}=\Delta \tau_{r m}$, and $\tilde{\tau}_{b r} \triangleq \Delta \tau_{b r}$. 


$$
\mathbf{J}_{\mathbf{p}_{\mathrm{M}}}=\left[\begin{array}{ccccccccc}
\nabla_{x_{\mathrm{M}}} \rho_{\mathrm{BM}} & \nabla_{x_{\mathrm{M}}} \theta_{\mathrm{BM}} & \nabla_{x_{\mathrm{M}}} \phi_{\mathrm{BM}} & \nabla_{x_{\mathrm{M}}} \tau_{\mathrm{BM}} & \nabla_{x_{\mathrm{M}}} \rho_{\mathrm{BRM}} & \nabla_{x_{\mathrm{M}}} \theta_{\mathrm{RM}} & \nabla_{x_{\mathrm{M}}} \phi_{\mathrm{RM}} & \nabla_{x_{\mathrm{M}}} \tau_{\mathrm{RM}} \\
\nabla_{y_{\mathrm{M}}} \rho_{\mathrm{BM}} & \nabla_{y_{\mathrm{M}}} \theta_{\mathrm{BM}} & \nabla_{y_{\mathrm{M}}} \phi_{\mathrm{BM}} & \nabla_{y_{\mathrm{M}}} \tau_{\mathrm{BM}} & \nabla_{y_{\mathrm{M}}} \rho_{\mathrm{BRM}} & \nabla_{y_{\mathrm{M}}} \theta_{\mathrm{RM}} & \nabla_{y_{\mathrm{M}}} \phi_{\mathrm{RM}} & \nabla_{y_{\mathrm{M}}} \tau_{\mathrm{RM}} \\
\nabla_{z_{\mathrm{M}}} \rho_{\mathrm{BM}} & \nabla_{z_{\mathrm{M}}} \theta_{\mathrm{BM}} & \nabla_{z_{\mathrm{M}}} \phi_{\mathrm{BM}} & \nabla_{z_{\mathrm{M}}} \tau_{\mathrm{BM}} & \nabla_{z_{\mathrm{M}}} \rho_{\mathrm{BRM}} & \nabla_{z_{\mathrm{M}}} \theta_{\mathrm{RM}} & \nabla_{z_{\mathrm{M}}} \phi_{\mathrm{BM}} & \nabla_{z_{\mathrm{M}}} \tau_{\mathrm{RM}}
\end{array}\right],
$$

Two-stage localization: The derivatives for the indirect approach can be found now as

$$
\begin{aligned}
& \nabla_{\rho_{\mathrm{BM}}} \mu_{b}[n]=\mu_{b, \mathrm{BM}}[n] / \rho_{\mathrm{BM}}, \nabla_{\rho_{\mathrm{BRM}}} \mu_{b}[n]=\mu_{b, \mathrm{BRM}}[n] / \rho_{\mathrm{BRM}}, \\
& \nabla_{\theta_{\mathrm{BM}}} \mu_{b}[n]=-j 2 \pi f_{n} \sqrt{P} \rho_{\mathrm{BM}} \sum_{m=1}^{N_{\mathrm{M}}} \mu_{b m}[n] \nabla_{\theta_{\mathrm{BM}}} \tau_{b m}, \\
& \nabla_{\theta_{\mathrm{RM}}} \mu_{b}[n]=-j 2 \pi f_{n} \sqrt{P} \rho_{\mathrm{BRM}} \sum_{m=1}^{N_{\mathrm{M}}} \sum_{r=1}^{N_{\mathrm{R}}} \mu_{b r m}[n] \nabla_{\theta_{\mathrm{RM}}} \tau_{r m}, \\
& \nabla_{\phi_{\mathrm{BM}}} \mu_{b}[n]=-j 2 \pi f_{n} \sqrt{P} \rho_{\mathrm{BM}} \sum_{m=1}^{N_{\mathrm{M}}} \mu_{b m}[n] \nabla_{\phi_{\mathrm{BM}}} \tau_{b m}, \\
& \nabla_{\phi_{\mathrm{RM}}} \mu_{b}[n]=-j 2 \pi f_{n} \sqrt{P} \rho_{\mathrm{BRM}} \sum_{m=1}^{N_{\mathrm{M}}} \sum_{r=1}^{N_{\mathrm{R}}} \mu_{b r m}[n] \nabla_{\phi_{\mathrm{RM}}} \tau_{r m}, \\
& \nabla_{\tau_{\mathrm{BM}}} \mu_{b}[n]=-j 2 \pi f_{n} \sqrt{P} \rho_{\mathrm{BM}} \sum_{m=1}^{N_{\mathrm{M}}} \mu_{b m}[n] \nabla_{\tau_{\mathrm{BM}}} \tau_{b m}, \\
& \nabla_{\tau_{\mathrm{RM}}} \mu_{b}[n]=-j 2 \pi f_{n} \sqrt{P} \rho_{\mathrm{BRM}} \sum_{m=1}^{N_{\mathrm{M}}} \sum_{r=1}^{N_{\mathrm{R}}} \mu_{b r m}[n] \nabla_{\tau_{\mathrm{RM}}} \tau_{r m},
\end{aligned}
$$

As regards the derivatives with respect to the rotational angles of the mobile, we have $\forall \phi_{M} \in\left\{\alpha_{M}, \beta_{M}, \gamma_{M}\right\}$

$$
\begin{aligned}
\nabla_{\boldsymbol{\phi}_{\mathrm{M}}} \mu_{b}[n]= & -j 2 \pi f_{n} \sqrt{P}\left(\rho_{\mathrm{BM}} \sum_{m=1}^{N_{\mathrm{M}}} \mu_{b m}[n] \nabla_{\boldsymbol{\phi}_{\mathrm{M}}} \tilde{\tau}_{b m}\right. \\
& \left.+\rho_{\mathrm{BRM}} \sum_{m=1}^{N_{\mathrm{M}}} \sum_{r=1}^{N_{\mathrm{R}}} \mu_{b r m}[n] \nabla_{\boldsymbol{\phi}_{\mathrm{M}}} \tilde{\tau}_{r m}\right)
\end{aligned}
$$

The derivatives of the TOAs with respect to the parameters can be written for each $S \in\{B, R\}$ and the corresponding antenna index $s \in\{b, r\}$ as

$$
\begin{aligned}
& \nabla_{\theta_{\mathrm{SM}}} \tau_{s m}=\frac{d_{\mathrm{SM}}}{c d_{s m}}\left[\left(x_{m}-x_{s}\right) \sin \theta_{\mathrm{SM}} \cos \phi_{\mathrm{SM}}+\right. \\
& \left.\quad+\left(y_{m}-y_{s}\right) \sin \theta_{\mathrm{SM}} \sin \phi_{\mathrm{SM}}-\left(z_{m}-z_{s}\right) \cos \theta_{\mathrm{SM}}\right] \\
& \nabla_{\phi_{\mathrm{SM}}} \tau_{s m}=\frac{d_{\mathrm{SM}}}{c d_{s m}}\left[\left(x_{m}-x_{s}\right) \cos \theta_{\mathrm{SM}} \sin \phi_{\mathrm{SM}}+\right. \\
& \left.\quad-\left(y_{m}-y_{s}\right) \cos \theta_{\mathrm{SM}} \cos \phi_{\mathrm{SM}}\right] \\
& \nabla_{\phi_{\mathrm{M}}} \tau_{s m}=\frac{1}{c d_{s m}}\left[x_{m} \nabla_{\phi_{\mathrm{M}}} x_{m}+y_{m} \nabla_{\phi_{\mathrm{M}}} y_{m}+z_{m} \nabla_{\phi_{\mathrm{M}}} z_{m}\right. \\
& \quad-\left(x_{s} \nabla_{\phi_{\mathrm{M}}} x_{m}+y_{s} \nabla_{\phi_{\mathrm{M}}} y_{m}+z_{s} \nabla_{\phi_{\mathrm{M}}} z_{m}+\right. \\
& \quad+d_{\mathrm{SM}}\left(\nabla_{\phi_{\mathrm{M}}} x_{m} \cos \theta_{\mathrm{BM}} \cos \phi_{\mathrm{BM}}+\right. \\
& \left.\left.\left.\quad+\nabla_{\phi_{\mathrm{M}}} y_{m} \cos \theta_{\mathrm{BM}} \sin \phi_{\mathrm{BM}}+\nabla_{\phi_{\mathrm{M}}} z_{m} \sin \theta_{\mathrm{BM}}\right)\right)\right] \\
& \nabla_{\tau_{\mathrm{SM}}} \tau_{s m}=\frac{1}{d_{s m}}\left[d_{\mathrm{SM}}-G_{s m}^{(2)}\right], \\
& \nabla_{\phi_{\mathrm{M}}} \Delta \tau_{s m}=\nabla_{\phi_{\mathrm{M}}} \tau_{s m}, \quad \nabla_{\theta_{\mathrm{SM}}} \Delta \tau_{s m}=\nabla_{\theta_{\mathrm{SM}}} \tau_{s m}, \\
& \nabla_{\phi_{\mathrm{SM}}} \Delta \tau_{s m}=\nabla_{\phi_{\mathrm{SM}}} \tau_{s m}, \quad \nabla_{\tau_{\mathrm{SM}}} \Delta \tau_{s m}=\nabla_{\tau_{\mathrm{SM}}} \tau_{s m}-1,
\end{aligned}
$$

where, by letting $c_{x_{\mathrm{M}}} \triangleq \cos \left(x_{\mathrm{M}}\right)$ and $s_{x_{\mathrm{M}}} \triangleq \sin \left(x_{\mathrm{M}}\right)$, we get

$$
\begin{aligned}
& \nabla_{\alpha_{\mathrm{M}}} x_{m}=-s_{\alpha_{\mathrm{M}}} c_{\beta_{\mathrm{M}}} x_{m}^{(0)}+\left[-s_{\alpha_{\mathrm{M}}} s_{\beta_{\mathrm{M}}} s_{\gamma_{\mathrm{M}}}-c_{\alpha_{\mathrm{M}}} c_{\gamma_{\mathrm{M}}}\right] y_{m}^{(0)} \\
& +\left[c_{\alpha_{\mathrm{M}}} s_{\gamma_{\mathrm{M}}}-s_{\alpha_{\mathrm{M}}} s_{\beta_{\mathrm{M}}} c_{\gamma_{\mathrm{M}}}\right] z_{m}^{(0)} \text {, } \\
& \nabla_{\beta_{\mathrm{M}}} x_{m}=-c_{\alpha_{\mathrm{M}}} s_{\beta_{\mathrm{M}}} x_{m}^{(0)}+c_{\alpha_{\mathrm{M}}} c_{\beta_{\mathrm{M}}} s_{\gamma_{\mathrm{M}}} y_{m}^{(0)}+c_{\alpha_{\mathrm{M}}} c_{\beta_{\mathrm{M}}} c_{\gamma_{\mathrm{M}}} z_{m}^{(0)} \text {, } \\
& \nabla_{\gamma_{\mathrm{M}}} x_{m}=\left[c_{\alpha_{\mathrm{M}}} s_{\beta_{\mathrm{M}}} c_{\gamma_{\mathrm{M}}}+s_{\alpha_{\mathrm{M}}} s_{\gamma_{\mathrm{M}}}\right] y_{m}^{(0)}+ \\
& +\left[s_{\alpha_{\mathrm{M}}} c_{\gamma_{\mathrm{M}}}-c_{\alpha_{\mathrm{M}}} s_{\beta_{\mathrm{M}}} s_{\gamma_{\mathrm{M}}}\right] z_{m}^{(0)} \\
& \nabla_{\alpha_{\mathrm{M}}} y_{m}=c_{\alpha_{\mathrm{M}}} c_{\beta_{\mathrm{M}}} x_{m}^{(0)}+\left[-c_{\gamma_{\mathrm{M}}} s_{\alpha_{\mathrm{M}}}+c_{\alpha_{\mathrm{M}}} s_{\beta_{\mathrm{M}}} s_{\gamma_{\mathrm{M}}}\right] y_{m}^{(0)}+ \\
& +\left[c_{\gamma_{\mathrm{M}}} c_{\alpha_{\mathrm{M}}} s_{\beta_{\mathrm{M}}}+s_{\alpha_{\mathrm{M}}} s_{\gamma_{\mathrm{M}}}\right] z_{m}^{(0)} \\
& \nabla_{\beta_{\mathrm{M}}} y_{m}=-s_{\alpha_{\mathrm{M}}} s_{\beta_{\mathrm{M}}} x_{m}^{(0)}+s_{\alpha_{\mathrm{M}}} c_{\beta_{\mathrm{M}}} s_{\gamma_{\mathrm{M}}} y_{m}^{(0)}+c_{\gamma_{\mathrm{M}}} s_{\alpha_{\mathrm{M}}} c_{\beta_{\mathrm{M}}} z_{m}^{(0)} \text {, } \\
& \nabla_{\gamma_{\mathrm{M}}} y_{m}=\left[-s_{\gamma_{\mathrm{M}}} c_{\alpha_{\mathrm{M}}}+s_{\alpha_{\mathrm{M}}} s_{\beta_{\mathrm{M}}} c_{\gamma_{\mathrm{M}}}\right] y_{m}^{(0)}+ \\
& -\left[s_{\gamma_{\mathrm{M}}} s_{\alpha_{\mathrm{M}}} s_{\beta_{\mathrm{M}}}+c_{\alpha_{\mathrm{M}}} c_{\gamma_{\mathrm{M}}}\right] z_{m}^{(0)} \text {, } \\
& \nabla_{\alpha_{\mathrm{M}}} z_{m}=0 \text {, } \\
& \nabla_{\beta_{\mathrm{M}}} z_{m}=-c_{\beta_{\mathrm{M}}} x_{m}^{(0)}-s_{\beta_{\mathrm{M}}} s_{\gamma_{\mathrm{M}}} y_{m}^{(0)}-s_{\beta_{\mathrm{M}}} c_{\gamma_{\mathrm{M}}} z_{m}^{(0)} \text {, } \\
& \nabla_{\gamma_{\mathrm{M}}} z_{m}=c_{\beta_{\mathrm{M}}} c_{\gamma_{\mathrm{M}}} y_{m}^{(0)}-c_{\beta_{\mathrm{M}}} s_{\gamma_{\mathrm{M}}} z_{m}^{(0)} \text {. }
\end{aligned}
$$

Direct localization: When a direct localization approach is used, the signal can be rewritten as

$$
\mu_{b}=\sum_{m=1}^{N_{\mathrm{M}}}\left(f_{b m}\left(\rho_{\mathrm{BM}}, d_{b m}\right)+\sum_{r=1}^{N_{\mathrm{R}}} g_{b r m}\left(\rho_{\mathrm{BRM}}, d_{r m}\right)\right)
$$

where $f_{b m}$ and $g_{b r m}$ are two non-linear functions depending on the parameters to be estimated, ${ }^{5}$ defined as ${ }^{6}$

$$
f_{b m}\left(\rho_{\mathrm{BM}}, d_{b m}\right) \triangleq \sqrt{P} x_{m}[n] \rho_{\mathrm{BM}}\left(\mathbf{p}_{\mathrm{M}}\right) \exp \left(-j 2 \pi \frac{f_{n}}{c} \tilde{d}_{b m}\right)
$$

where $\tilde{d}_{b m}=\tilde{d}_{b m}\left(\mathbf{p}_{\mathrm{M}}, \boldsymbol{\phi}_{\mathrm{M}}\right) \triangleq d_{b m}$ and $\tilde{d}_{b m} \triangleq \Delta d_{b m}$ in synchronous and asynchronous cases, respectively, and

$$
\begin{aligned}
g_{b r m}\left(\rho_{\mathrm{BRM}}, d_{r m}\right) & \triangleq \sqrt{P} x_{m}[n] \rho_{\mathrm{BRM}}\left(\mathbf{p}_{\mathrm{M}}\right) \exp \left(j \theta_{r}\right) . \\
& \times \exp \left(-j 2 \pi \frac{f_{n}}{c}\left(\tilde{d}_{b r}+\tilde{d}_{r m}\right)\right),
\end{aligned}
$$

where $\tilde{d}_{r m} \triangleq d_{r m}$ and $\tilde{d}_{r m} \triangleq \Delta d_{r m}$ in synchronous scheme; whereas $\tilde{d}_{b r}=d_{b r}$ and $\tilde{d}_{b r}=\Delta d_{b r}$ in asynchronous scheme.

The gradient vector with respect to the position, $\mathbf{p}_{\mathbf{M}}$, and orientation, $\phi_{\mathrm{M}}$, can be written from (82) as

$$
\begin{aligned}
& \nabla_{\mathbf{p}_{\mathrm{M}}}\left(\mu_{b}\right)=\sum_{m=1}^{N_{\mathrm{M}}} \nabla_{\mathbf{p}_{\mathrm{M}}} f_{b m}+\sum_{r=1}^{N_{\mathrm{R}}} \nabla_{\mathbf{p}_{\mathrm{M}}} g_{b r m}, \\
& \nabla_{\boldsymbol{\phi}_{\mathrm{M}}}\left(\mu_{b}\right)=\sum_{m=1}^{N_{\mathrm{M}}} \nabla_{\boldsymbol{\phi}_{\mathrm{M}}} f_{b m}+\sum_{r=1}^{N_{\mathrm{R}}} \nabla_{\boldsymbol{\phi}_{\mathrm{M}}} g_{b r m},
\end{aligned}
$$

\footnotetext{
${ }^{5}$ Generally, the optimal design of RIS phases can depend on the UE location and orientation. For convenience, such a dependence is neglected in (82).

${ }^{6}$ Here we have dropped the synchronization mismatches and array errors.
} 
where for the direct path we have

$$
\begin{aligned}
\nabla_{\mathbf{p}_{\mathrm{M}}} f_{b m}= & \sqrt{P} x_{m}\left[\nabla_{d_{\mathrm{BM}}} \rho_{\mathrm{BM}} \nabla_{\mathbf{p}_{\mathrm{M}}} d_{\mathrm{BM}}+\right. \\
& \left.-j 2 \pi \frac{f_{n}}{c} \rho_{\mathrm{BM}} \nabla_{\mathbf{p}_{\mathrm{M}}} \tilde{d}_{b m}\right] e^{-j 2 \pi \frac{f_{n}}{c} \tilde{d}_{b m}}, \\
\nabla_{\boldsymbol{\phi}_{\mathrm{M}}} f_{b m}= & -j 2 \pi \frac{f_{n}}{c} \sqrt{P} \rho_{\mathrm{BM}} x_{m} e^{-j 2 \pi \frac{f_{n}}{c} \nabla_{\phi_{\mathrm{M}}} \tilde{d}_{b m}},
\end{aligned}
$$

while for the RIS-relayed path it is

$$
\begin{aligned}
\nabla_{\mathbf{p}_{\mathrm{M}}} g_{b r m}= & \sqrt{P} x_{m} \exp \left(j \theta_{r}\right)\left[\nabla_{d_{r m}} \rho_{\mathrm{BRM}} \nabla_{\mathbf{p}_{\mathrm{M}}} d_{\mathrm{RM}}+\right. \\
& \left.-j 2 \pi \frac{f_{n}}{c} \rho_{\mathrm{BRM}} \nabla_{\mathbf{p}_{\mathrm{M}}} \tilde{d}_{r m}\right] e^{-j 2 \pi \frac{f_{n}}{c}\left(\tilde{d}_{r m}+\tilde{d}_{b r}\right)}, \\
\nabla_{\boldsymbol{\phi}_{\mathrm{M}}} g_{b r m}= & -\frac{j 2 \pi f_{n}}{c} \sqrt{P} \rho_{\mathrm{BRM}} x_{m} e^{j \theta_{r}} e^{-j 2 \pi \frac{f_{n}}{c}\left(\tilde{d}_{r m}+\tilde{d}_{b r}\right)}, \\
& \times \nabla_{\boldsymbol{\phi}_{\mathrm{M}}} \tilde{d}_{r m},
\end{aligned}
$$

The derivatives of the path-loss amplitudes with respect to the distances between array centers are

$$
\nabla_{d_{\mathrm{BM}}} \rho_{\mathrm{BM}}=-\frac{\lambda}{4 \pi d_{\mathrm{BM}}^{2}}, \quad \nabla_{d_{\mathrm{RM}}} \rho_{\mathrm{BRM}}=-\frac{\lambda}{4 \pi\left(d_{\mathrm{BR}}+d_{\mathrm{RM}}\right)^{2}} .
$$

By denoting with $a_{\mathrm{M}} \in\left\{x_{\mathrm{M}}, y_{\mathrm{M}}, z_{\mathrm{M}}\right\}, \mathrm{S} \in\{\mathrm{B}, \mathrm{R}\}$ and $s \in$ $\{b, r\}$, we can obtain

$$
\begin{aligned}
& \nabla_{a_{\mathrm{M}}} \Delta d_{s m}=\nabla_{a_{\mathrm{M}}} d_{s m}-\nabla_{a_{\mathrm{M}}} d_{\mathrm{SM}} \\
& \nabla_{a_{\mathrm{M}}} d_{s m}=\frac{d_{\mathrm{SM}}}{d_{s m}}\left(\nabla_{a_{\mathrm{M}}} d_{\mathrm{SM}}-\frac{\nabla_{a_{\mathrm{M}}} d_{\mathrm{SM}}}{d_{\mathrm{SM}}} \mathrm{G}_{s m}^{(2)}-\nabla_{a_{\mathrm{M}}} \mathrm{G}_{s m}^{(2)}\right),
\end{aligned}
$$

where $\nabla_{a_{\mathrm{M}}} d_{\mathrm{SM}}\left(a_{\mathrm{M}}\right)=\frac{a_{\mathrm{M}}-a_{\mathrm{S}}}{d_{\mathrm{SM}}}$ and where

$$
\begin{aligned}
\nabla_{a_{\mathrm{M}} \mathrm{G}_{s m}^{(2)}=} & -\left(x_{m}-x_{s}\right) \sin \left(\theta_{\mathrm{SM}}\right) \cos \left(\phi_{\mathrm{SM}}\right) \nabla_{a_{\mathrm{M}}} \theta_{\mathrm{SM}} \\
& -\left(x_{m}-x_{s}\right) \cos \left(\theta_{\mathrm{SM}}\right) \sin \left(\phi_{\mathrm{SM}}\right) \nabla_{a_{\mathrm{M}}} \phi_{\mathrm{SM}} \\
& -\left(y_{m}-y_{s}\right) \sin \left(\theta_{\mathrm{SM}}\right) \sin \left(\phi_{\mathrm{SM}}\right) \nabla_{a_{\mathrm{M}}} \theta_{\mathrm{SM}} \\
& +\left(y_{m}-y_{s}\right) \cos \left(\theta_{\mathrm{SM}}\right) \cos \left(\phi_{\mathrm{SM}}\right) \nabla_{a_{\mathrm{M}}} \phi_{\mathrm{SM}} \\
& +\left(z_{m}-z_{s}\right) \cos \left(\theta_{\mathrm{SM}}\right) \nabla_{a_{\mathrm{M}}} \theta_{\mathrm{SM}}
\end{aligned}
$$

with $\nabla_{a_{\mathrm{M}}} \phi_{\mathrm{SM}}$ and $\nabla_{a_{\mathrm{M}}} \theta_{\mathrm{SM}}$ as in Appendix A. Thus, the derivatives in (92) becomes

$$
\begin{aligned}
& \nabla_{x_{\mathrm{M}}} \mathrm{G}_{s m}^{(2)}=\frac{x_{m}-x_{s}}{d_{\mathrm{SM}}}\left[\sin ^{2} \theta_{\mathrm{SM}} \cos ^{2} \phi_{\mathrm{SM}}+\sin ^{2} \phi_{\mathrm{SM}}\right] \\
& +\frac{y_{m}-y_{s}}{d_{\mathrm{SM}}}\left[\sin ^{2} \theta_{\mathrm{SM}} \cos \phi_{\mathrm{SM}} \sin \phi_{\mathrm{SM}}-\sin \phi_{\mathrm{SM}} \cos \phi_{\mathrm{SM}}\right] \\
& \quad-\frac{z_{m}-z_{s}}{d_{\mathrm{SM}}} \sin \theta_{\mathrm{SM}} \cos \phi_{\mathrm{SM}} \cos \theta_{\mathrm{SM}} \\
& \nabla_{\mathrm{yM}_{\mathrm{M}}} \mathrm{G}_{s m}^{(2)}=\frac{x_{m}-x_{s}}{d_{\mathrm{SM}}}\left[\sin ^{2} \theta_{\mathrm{SM}} \sin \phi_{\mathrm{SM}} \cos \phi_{\mathrm{SM}}\right. \\
& \left.\quad-\sin \phi_{\mathrm{SM}} \cos \phi_{\mathrm{SM}}\right]+\frac{y_{m}-y_{s}}{d_{\mathrm{SM}}}\left[\sin ^{2} \theta_{\mathrm{SM}} \sin ^{2} \phi_{\mathrm{SM}}\right. \\
& \left.\quad+\cos ^{2} \phi_{\mathrm{SM}}\right]-\frac{z_{m}-z_{s}}{d_{\mathrm{SM}}}\left[\sin \theta_{\mathrm{SM}} \cos \theta_{\mathrm{SM}} \sin \phi_{\mathrm{SM}}\right]
\end{aligned}
$$

$$
\begin{gathered}
\nabla_{\mathrm{z}_{\mathrm{M}}} \mathrm{G}_{s m}^{(2)}=-\frac{x_{m}-x_{s}}{d_{\mathrm{BM}}} \sin \theta_{\mathrm{SM}} \cos \theta_{\mathrm{SM}} \cos \phi_{\mathrm{SM}} \\
+\frac{y_{m}-y_{s}}{d_{\mathrm{SM}}}\left[\sin \theta_{\mathrm{SM}} \cos \theta_{\mathrm{SM}} \sin \phi_{\mathrm{SM}}\right] \\
+\frac{z_{m}-z_{s}}{d_{\mathrm{SM}}} \cos ^{2} \theta_{\mathrm{SM}} .
\end{gathered}
$$

\section{REFERENCES}

[1] M. Di Renzo et al., "Smart radio environments empowered by reconfigurable AI meta-surfaces: an idea whose time has come," EURASIP J. Wireless Commun. Net., vol. 2019, no. 1, pp. 1-20, May 2019.

[2] Q. Nadeem et al., "Intelligent reflecting surface assisted multi-user MISO communication: Channel estimation and beamforming design," IEEE Open J. Commun., pp. 1-1, 2020.

[3] M. Bennis et al., "Ultrareliable and low-latency wireless communication: Tail, risk, and scale," Proc. IEEE, vol. 106, no. 10, pp. 1834-1853, 2018.

[4] B. Zong et al., " $6 \mathrm{G}$ technologies: Key drivers, core requirements, system architectures, and enabling technologies," IEEE Veh. Technol. Mag., vol. 14 , no. 3, pp. 18-27, 2019.

[5] A. D. J. Torres et al., "Cramér-Rao bounds for near-field localization," arXiv preprint arXiv:2104.14825, 2021.

[6] H. Sarieddeen et al., "Next generation Terahertz communications: A rendezvous of sensing, imaging, and localization," IEEE Comm. Maga., vol. 58, no. 5, pp. 69-75, June 2020.

[7] A. Taha et al., "Deep learning for large intelligent surfaces in millimeter wave and massive MIMO systems," in Proc. IEEE Global Commun. Conf. (GLOBECOM), 2019, pp. 1-6.

[8] E. Basar et al., "Wireless communications through reconfigurable intelligent surfaces," IEEE Access, vol. 7, pp. 116753-116 773, Aug. 2019.

[9] D. R. Smith et al., "Composite medium with simultaneously negative permeability and permittivity," Physical Review Lett., vol. 84, no. 18, p. 4184, May 2000.

[10] — "Metamaterials and negative refractive index," Science, vol. 305, no. 5685, pp. 788-792, 2004.

[11] N. Engheta and R. W. Ziolkowski, Metamaterials: Physics and Engineering Explorations. John Wiley \& Sons, 2006.

[12] N. Kaina et al., "Shaping complex microwave fields in reverberating media with binary tunable metasurfaces," Scientific reports, vol. 4, no. 1, pp. 1-8, 2014.

[13] P. del Hougne et al., "Optimally diverse communication channels in disordered environments with tuned randomness," Nature Electronics, vol. 2, no. 1, pp. 36-41, 2019.

[14] C. L. Holloway et al., "An overview of the theory and applications of metasurfaces: The two-dimensional equivalents of metamaterials," IEEE Antennas Propag. Mag., vol. 54, no. 2, pp. 10-35, Apr. 2012.

[15] N. Shlezinger et al., "Dynamic metasurface antennas for uplink massive MIMO systems," IEEE Trans. Commun., vol. 67, no. 10, pp. 6829-6843, Oct. 2019.

[16] I. Yoo et al., "Enhancing capacity of spatial multiplexing systems using reconfigurable cavity-backed metasurface antennas in clustered MIMO channels," IEEE Trans. Commun., vol. 67, no. 2, pp. 1070-1084, Feb. 2018.

[17] H. Wang et al., "Dynamic metasurface antennas for MIMO-OFDM receivers with bit-limited ADCs," IEEE Trans. Commun., vol. 69, no. 4, pp. 2643-2659, April 2021

[18] D. Dardari, "Communicating with large intelligent surfaces: Fundamental limits and models," IEEE J. Sel. Areas Commun., 2020.

[19] D. Dardari and D. Massari, "Using metaprisms for performance improvement in wireless communications," IEEE Trans. Wireless Commun., vol. 20, no. 5, May 2021.

[20] C. Huang et al., "Holographic MIMO surfaces for $6 \mathrm{G}$ wireless networks: Opportunities, challenges, and trends," IEEE Wireless Commun., vol. 27, no. 5, pp. 118-125, Oct. 2020.

[21] Q. Nadeem et al., "Asymptotic max-min SINR analysis of reconfigurable intelligent surface assisted miso systems," IEEE Trans. Wireless Commun., pp. 1-1, 2020.

[22] M. A. Kishk and M.-S. Alouini, "Exploiting randomly-located blockages for large-scale deployment of intelligent surfaces," IEEE J. Sel. Areas Commun., vol. 39, no. 4, pp. 1043-1056, April 2021.

[23] A. Kafizov et al., "Wireless network coding with intelligent reflecting surfaces," IEEE Commun. Lett., 2021, [Early Access].

[24] Q. Wu and R. Zhang, "Towards smart and reconfigurable environment: Intelligent reflecting surface aided wireless network," IEEE Commun. Mag., vol. 58, no. 1, pp. 106-112, 2020.

[25] B. M. Masini et al., "The use of meta-surfaces in vehicular networks," J. Sens. Actuator Netw., vol. 9, no. 1, p. 15, 2020.

[26] L. Yang et al., "On the performance of RIS-assisted dual-hop UAV communication systems," IEEE Trans. Veh. Technol., pp. 1-1, June 2020.

[27] L. Zhang et al., "Intelligent reflecting surface aided MIMO cognitive radio systems," IEEE Trans. Veh. Technol., vol. 69, no. 10, pp. 11445 11457 , Oct. 2020. 
[28] A. Elzanaty et al., "5G and EMF exposure: Misinformation, open questions, and potential solutions," Frontiers in Communications and Networks, 2021.

[29] L. Chiaraviglio et al., "Health risks associated with 5G exposure: A view from the communications engineering perspective," arXiv preprint arXiv:2006.00944, 2020.

[30] H. Ibraiwish et al., "EMF-aware cellular networks in RIS-assisted environments," KAUST Repository, 2021.

[31] R. Alghamdi et al., "Intelligent surfaces for 6G wireless networks: A survey of optimization and performance analysis techniques," IEEE Access, vol. 8, pp. 202 795-202 818, Oct. 2020.

[32] H. Wymeersch et al., "Radio localization and mapping with reconfigurable intelligent surfaces: Challenges, opportunities, and research directions," IEEE Veh. Technol. Mag., vol. 15, no. 4, pp. 52-61, Dec. 2020.

[33] J. He et al., "Large intelligent surface for positioning in millimeter wave MIMO systems," in Proc. IEEE Vehic. Tech. Conf. (VTC), May 2020, pp. 1-5.

[34] S. M. Razavizadeh et al., "Three-dimensional beamforming: A new enabling technology for 5G wireless networks," IEEE Signal Process. Mag., vol. 31, no. 6, pp. 94-101, 2014.

[35] S. K. Moghaddam and S. M. Razavizadeh, "Joint tilt angle adaptation and beamforming in multicell multiuser cellular networks," Computers \& Electrical Engineering, vol. 61, pp. 195-207, 2017.

[36] S. M. Razavizadeh and T. Svensson, "3D beamforming in reconfigurable intelligent surfaces-assisted wireless communication networks," pp. 1-5, 2020.

[37] Y. Shen and M. Z. Win, "On the accuracy of localization systems using wideband antenna arrays," IEEE Trans. Commun., vol. 58, no. 1, pp. 270-280, 2010.

[38] A. Guerra et al., "Single anchor localization and orientation performance limits using massive arrays: MIMO vs. beamforming," IEEE Trans. Wireless Commun., vol. 17, no. 8, pp. 5241-5255, 2018.

[39] — , "Near-field tracking with large antenna arrays: Fundamental limits and practical algorithms," IEEE Trans. Signal Process., In publication, 2021.

[40] A. Guerra et al., "Position and orientation error bound for wideband massive antenna arrays," in Proc. IEEE Int. Conf. Commun. Workshop, 2015, pp. 853-858.

[41] Y. Han et al., "Performance limits and geometric properties of array localization," IEEE Trans. Inf. Th., vol. 62, no. 2, pp. 1054-1075, 2015.

[42] Y. Wang et al., "On the resolution limits for MIMO localization," IEEE Commun. Lett., vol. 23, no. 3, pp. 462-465, Mar. 2019.

[43] F. Guidi et al., "Indoor environment-adaptive mapping with beamsteering massive arrays," IEEE Trans. Veh. Technol., vol. 67, no. 10, pp. $10139-10143,2018$.

[44] Y. Wang et al., "Joint spatiotemporal multipath mitigation in large-scale array localization," IEEE Trans. Signal Process., vol. 67, no. 3, pp. 783797, 2019.

[45] W. Roh et al., "Millimeter-wave beamforming as an enabling technology for $5 \mathrm{G}$ cellular communications: theoretical feasibility and prototype results," IEEE Commun. Mag., vol. 52, no. 2, pp. 106-113, 2014.

[46] F. Guidi et al., "Personal mobile radars with millimeter-wave massive arrays for indoor mapping," IEEE Trans. Mobile Comput., vol. 15, no. 6 , pp. 1471-1484, Jun. 2016.

[47] K. Witrisal et al., "High-accuracy localization for assisted living: 5G systems will turn multipath channels from foe to friend," IEEE Signal Process. Mag., vol. 33, no. 2, pp. 59-70, 2016.

[48] F. Guidi et al., "Joint energy detection and massive array design for localization and mapping," IEEE Trans. Wireless Commun., vol. 16, no. 3, pp. 1359-1371, Mar. 2017.

[49] S. Hu et al., "Beyond massive MIMO: The potential of data transmission with large intelligent surfaces," IEEE Trans. Signal Process., vol. 66, no. 10, May 2018.

[50] J. He et al., "Adaptive beamforming design for mmWave RIS-aided joint localization and communication," in Proc. IEEE Wireless Commu. Netw. Conf. Work. (WCNCW), June 2020, pp. 1-6.

[51] S. Hu et al., "Beyond massive MIMO: The potential of positioning with large intelligent surfaces," IEEE Trans. Signal Process., vol. 66, no. 7, pp. 1761-1774, 2018.

[52] H. Zhang et al., "Towards ubiquitous positioning by leveraging reconfigurable intelligent surface," IEEE Commun. Lett., vol. 25, no. 1, pp. 284-288, Jan. 2021.

[53] F. Guidi and D. Dardari, "Radio positioning with EM processing of the spherical wavefront," IEEE Trans. on Wireless Commun., vol. 20, no. 6, pp. 3571-3586, June 2021.
[54] E. Björnson and L. Sanguinetti, "Power scaling laws and near-field behaviors of massive MIMO and intelligent reflecting surfaces," IEEE Open J. Commun., vol. 1, pp. 1306-1324, Sept. 2020.

[55] P. Nepa and A. Buffi, "Near-field-focused microwave antennas: Nearfield shaping and implementation," IEEE Antennas Propag. Mag., vol. 59, no. 3, pp. 42-53, Jun. 2017

[56] S. M. LaValle, Planning algorithms. Cambridge university press, 2006.

[57] A. Shahmansoori et al., "Position and Orientation Estimation Through Millimeter-Wave MIMO in 5G Systems," IEEE Trans. Wireless Commun., vol. 17, no. 3, pp. 1822-1835, 2018

[58] H. L. Van Trees, Classical Detection and Estimation Theory. John Wiley \& Sons, Ltd, 2002, ch. 2, pp. 19-165.

[59] Y. Shen and M. Z. Win, "Fundamental limits of wideband localizationpart I: A general framework," IEEE Trans. Inf. Th., vol. 56, no. 10, pp. 4956-4980, 2010.

[60] S. Gezici, "A survey on wireless position estimation," Wireless personal communications, vol. 44, no. 3, pp. 263-282, 2008.

[61] S. M. Kay, Fundamentals of statistical signal processing. Prentice Hall PTR, 1993

[62] C. A. Balanis, Antenna theory: analysis and design. Wiley-Interscience, 2005.

[63] Z. Yang et al., "Off-grid direction of arrival estimation using sparse Bayesian inference," IEEE Trans. Signal Process., vol. 61, no. 1, pp. $38-43$, Oct. 2013.

[64] A. Elzanaty et al., "Limits on sparse data acquisition: RIC analysis of finite Gaussian matrices," IEEE Trans. Inf. Th., vol. 65, no. 3, pp. 15781588, 2019.

[65] K. Lee et al., "Subspace methods for joint sparse recovery," IEEE Trans. Inf. Th., vol. 58, no. 6, pp. 3613-3641, Feb. 2012.

[66] A. Elzanaty et al., "Weak RIC analysis of finite Gaussian matrices for joint sparse recovery," IEEE Signal Process. Lett., vol. 24, no. 10, pp. 1473-1477, 2017.

[67] D. Dardari et al., "Threshold-based time-of-arrival estimators in UWB dense multipath channels," IEEE Trans. Comm., vol. 56, no. 8, pp. $1366-$ 1378, Aug. 2008

[68] — "Ranging with ultrawide bandwidth signals in multipath environments," Proc. IEEE, vol. 97, no. 2, pp. 404-426, Mar. 2009.

[69] A. Giorgetti and M. Chiani, "Time-of-arrival estimation based on information theoretic criteria," IEEE Trans. Signal Process., vol. 61, no. 8, pp. 1869-1879, Jan. 2013.

[70] D. Dardari et al., "Indoor tracking: Theory, methods, and technologies," IEEE Trans. Veh. Technol., vol. 64, no. 4, pp. 1263-1278, Feb. 2015.

[71] R. Yarlagadda et al., "GPS GDOP metric," IEE Proc. - Radar, sonar, nav., vol. 147, no. 5, pp. 259-264, 2000.

[72] N. Levanon, "Lowest GDOP in 2-D scenarios," IEE Proc. - Radar, Sonar, Nav., vol. 147, no. 3, pp. 149-155, 2000.

[73] Q. Wu and R. Zhang, "Intelligent Reflecting Surface Enhanced Wireless Network via Joint Active and Passive Beamforming," IEEE Trans. Wireless Commun., vol. 18, no. 11, pp. 5394-5409, 2019.

[74] J. MacQueen, "Some methods for classification and analysis of multivariate observations," in Proc. of the Fifth Berkeley Symp. on Math. Stat. and Prob., Volume 1: Stat. Berkeley, Calif.: University of California Press, 1967, pp. 281-297.

[75] S. Boyd and L. Vandenberghe, Convex optimization. Cambridge university press, 2004.

[76] Z. Zhou et al., "Joint Transmit Precoding and Reconfigurable Intelligent Surface Phase Adjustment: A Decomposition-Aided Channel Estimation Approach," IEEE Trans. Commun., vol. 69, no. 2, pp. 1228-1243, 2021.

[77] J. An et al., "Optimal Pilot Power Based Channel Estimation Improves the Throughput of Intelligent Reflective Surface Assisted Systems," IEEE Trans. Veh. Tech., vol. 69, no. 12, pp. 16202-16206, 2020.

[78] A. Elzanaty et al., "Towards 6G holographic localization: Enabling technologies and perspectives," arXiv preprint arXiv:2103.12415, 2021.

[79] W. Hong et al., "Millimeter-wave 5G antennas for smartphones: Overview and experimental demonstration," IEEE Trans. Antennas Propag., vol. 65, no. 12, pp. 6250-6261, 2017.

[80] Q. Nadeem et al., "Elevation beamforming with full dimension MIMO architectures in 5G systems: A tutorial," IEEE Commun. Surveys Tuts., vol. 21, no. 4, pp. 3238-3273, 2019.

[81] A. Zaidi et al., "Chapter 2 - NR physical layer: Overview," in $5 G$ Physical Layer. Academic Press, 2018, pp. 21 - 34.

[82] M. Kang and M.-S. Alouini, "A comparative study on the performance of MIMO MRC systems with and without cochannel interference," IEEE Trans. Commun., vol. 52, no. 8, pp. 1417-1425, 2004.

[83] Y. Yang et al., "Intelligent reflecting surface meets OFDM: Protocol design and rate maximization," IEEE Trans. Commun., vol. 68, no. 7, pp. $4522-4535,2020$ 